\title{
Probabilistic assignment: an extension approach
}

Link to publication record in Manchester Research Explorer

\section{Citation for published version (APA):}

Cho, W. J. (2013). Probabilistic assignment: an extension approach.

\section{Citing this paper}

Please note that where the full-text provided on Manchester Research Explorer is the Author Accepted Manuscript or Proof version this may differ from the final Published version. If citing, it is advised that you check and use the publisher's definitive version.

\section{General rights}

Copyright and moral rights for the publications made accessible in the Research Explorer are retained by the authors and/or other copyright owners and it is a condition of accessing publications that users recognise and abide by the legal requirements associated with these rights.

\section{Takedown policy}

If you believe that this document breaches copyright please refer to the University of Manchester's Takedown Procedures [http://man.ac.uk/04Y6Bo] or contact uml.scholarlycommunications@manchester.ac.uk providing relevant details, so we can investigate your claim.

\section{OPEN ACCESS}




\title{
Probabilistic Assignment: An Extension Approach*
}

\author{
Wonki Jo Cho ${ }^{\dagger}$
}

July 14, 2014

\begin{abstract}
We study the problem of allocating objects using lotteries when agents only submit preferences over objects. A standard approach is to "extend" agents' preferences over objects to preferences over lotteries, using (first-order) stochastic dominance, or the $s d$-extension. To better understand the role the $s d$-extension plays in analysis, we complement this approach with two alternatives to the $s d$-extension: the downward lexicographic extension, or the $d l$-extension, which lexicographically maximizes probabilities for preferred objects; and the upward lexicographic extension, or the ul-extension, which lexicographically minimizes probabilities for less preferred objects. We show that for each $e \in\{s d, d l, u l\}, e$-strategy-proofness (the strategy-proofness notion based on extension $e$ ) is equivalent to each of the following strategic properties: (i) e-adjacent strategy-proofness, which requires that no agent gain by switching the rankings of two adjacent objects; and (ii) e-lie monotonicity, which requires that the welfare of each agent weakly decrease as he reports increasingly bigger lies. These results imply that $d l$ - and $u l$-strategy-proofness together are sufficient for sd-strategy-proofness. We also show that $s d$-, $d l$-, and ul-efficiency are equivalent, and provide a generalization of the serial rule.
\end{abstract}

Journal of Economic Literature Classification Numbers: C70, C78, D61, D63

Key Words: probabilistic assignment; $e$-strategy-proofness; $e$-adjacent strategy-proofness; $e$ lie monotonicity; e-efficiency; generalized serial rules; random priority rule

*First draft: February 24, 2011. This paper is based on a chapter in my Ph.D. dissertation at the University of Rochester. I am grateful to William Thomson for his guidance and encouragement. I thank Guillaume Haeringer for his helpful comments after my presentation in the 2011 Society for Economic Design Conference, after which the notion of lie monotonicity and the related results were developed. I also thank Paulo Barelli, Srihari Govindan, Biung-Ghi Ju, Asen Kochov, Hervé Moulin, Romans Pancs, Jay Sethuraman, and seminar participants at Korea University, Seoul National University, UC Riverside, Hitotsubashi University, Sogang University, the 2011 Society for Economic Design Conference, and the 23rd International Conference on Game Theory for their comments.

${ }^{\dagger}$ School of Social Sciences, University of Manchester, Oxford Road, Manchester, M13 9PL, United Kingdom; jo.cho@manchester.ac.uk. 


\section{Introduction}

We study the problem of allocating indivisible commodities called objects using lotteries. Each agent has strict preferences over objects and ex post receives exactly one object. Going beyond deterministic assignments to probabilistic ones affords the advantage of converting objects into de facto perfectly divisible commodities. This raises the hope for fair allocation, which might have been impossible were only deterministic assignments considered. Due in large part to the latter fact, lotteries are frequently used in real life. Examples include on-campus housing allocation in colleges and student placement in public schools.

This problem is known as "probabilistic assignment", and we focus on the ordinal approach to the problem (Bogomolnaia and Moulin, 2001).1] According to the ordinal approach, agents submit their preferences over objects, and an assignment is selected based on this information only. But what agents receive cannot be directly evaluated according to the elicited preferences, and therefore, we cannot properly speak of properties of assignment rules. Bogomolnaia and Moulin (2001) circumvent this problem by "extending" preferences over objects to preferences over lotteries using (first-order) stochastic dominance $2^{2}$ We refer to this procedure as the $s d$-extension.

Once we adopt the $s d$-extension, it is automatically embedded in properties of assignment rules and affects their content. For instance, consider strategy-proofness, the requirement that no agent ever gain from misrepresenting his preferences. The notion of strategy-proofness based on the sd-extension, which we call sd-strategy-proofness, says that for each agent, the lottery he obtains by reporting his preferences truthfully should stochastically dominate any lottery he obtains by lying. Thus, the $s d$-extension plays a key role in this definition and more generally in the ordinal approach, but nevertheless, that role has not been much investigated so far. Since Bogomolnaia and Moulin (2001), most subsequent papers use the $s d$-extension (Che and Kojima, 2010; Haeringer and Halaburda, 2014; Hashimoto et al., 2014; Katta and Sethuraman, 2006; Kesten, 2009; Kojima, 2009; Liu and Pycia, 2012; and Yilmaz, 2009 and 2010) but they do not explore how that choice affects analysis.$^{3}$ In a departure from this practice, we consider two alternatives to the $s d$-extension and use all three of them in parallel to examine properties of assignment rules.

An extension is a mapping from preferences over objects to preferences over lotteries. Thus, the $s d$-extension is an example. The two alternative extensions we consider are related to lexicographic preferences (Hausner, 1954; Chipman, 1960). First is the "downward lexicographic" extension,

\footnotetext{
${ }^{1}$ Another approach is the cardinal one, which allows agents to express their von Neumann-Morgenstern preferences over lotteries (Hylland and Zeckhauser, 1979).

${ }^{2}$ While Bogomolnaia and Moulin (2001) are the first to use the $s d$-extension in the context of probabilistic assignment, it was adopted much earlier in probabilistic public choice. For instance, Gibbard (1977), in effect, applies the $s d$-extension in defining strategy-proofness.

${ }^{3}$ Only recently have some authors started to study probabilistic assignment with lexicographic preferences (Schulman and Vazirani, 2012; Bogomolnaia, 2012; Saban and Sethuraman, 2013).
} 

(a) sd-strategy-proofness $\Longleftrightarrow$ sd-adjacent strategy-proofness $\Longleftrightarrow$ sd-lie monotonicity

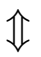 dl-adjacent strategy-proofness + ul-adjacent strategy-proofness

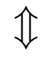 $d l$-strategy-proofness + ul-strategy-proofness

(b) dl-strategy-proofness $\Longleftrightarrow d l$-adjacent strategy-proofness $\Longleftrightarrow d l$-lie monotonicity

(c) ul-strategy-proofness $\Longleftrightarrow u l$-adjacent strategy-proofness $\Longleftrightarrow u l$-lie monotonicity

(d) sd-efficiency $\Longleftrightarrow d l$-efficiency $\Longleftrightarrow u l$-efficiency

Figure 1: Summary of main results. Panels (a)-(c) summarize Theorems 1 , 3 and panel (d) Theorem 4.

or the $d l$-extension. The $d l$-extension associates with each preference relation over objects the following preference relation over lotteries. Lotteries are compared in a lexicographic fashion, starting from the probabilities for the most preferred object. Given two lotteries, the lottery that assigns a higher probability to the most preferred object is preferred. If the two probabilities are equal, the lottery that assigns a higher probability to the second most preferred object is preferred. If the two probabilities are equal again, the lottery that assigns a higher probability to the third most preferred object is preferred, and so on.

The other alternative is the "upward lexicographic" extension, or the $u l$-extension. The preference relation over lotteries obtained by the $u l$-extension performs lexicographic comparison in the opposite way. Given two lotteries, the lottery that assigns a lower probability to the least preferred object is preferred. If the two probabilities are equal, the lottery that assigns a lower probability to the second least preferred object is preferred, and so on.

The $s d-, d l-$, and $u l$-extensions define different preferences over lotteries. Therefore, each property of assignment rules now has three versions, prefixed by the extension chosen; e.g., for an arbitrary extension e, e-strategy-proofness. We study their logical relation and evaluate assignment rules according to these new criteria.

Our first set of results concern strategy-proofness and apply to any model where agents submit preferences over a finite set of objects and receive lotteries over objects; e.g., voting (Gibbard, 1977), school choice (Abdulkadiroğlu and Sönmez, 2003b), house allocation with existing tenants (Abdulkadiroğlu and Sönmez, 1999). Following Carroll (2012) and Sato (2013a), we consider a strategic property called e-adjacent strategy-proofness. It requires that no agent be better off reporting a preference relation obtained by switching only two objects that are adjacent in his true preference rankings. One would expect that the property is much weaker than e-strategy-proofness. However, we show that for each $e \in\{s d, d l, u l\}$, e-adjacent strategy-proofness is equivalent to 
e-strategy-proofness (Theorem 1; Figure 1 summarizes our results). We also identify sufficient conditions on the (preference) domain that ensure the equivalence.

In most real-life applications, designing e-strategy-proof rules turns out to be very demanding, and the equivalence of e-strategy-proofness and e-adjacent strategy-proofness makes this task more tractable. Recall that checking e-strategy-proofness involves comparing all lotteries that are obtained by lying. This means that when there are $n$ objects, for each agent $i$ and each profile of announcements of all agents but $i$, the mechanism designer need to compare $n$ ! lotteries. This factorial function increases very fast (faster than any polynomial or exponential function), and just with 15 objects, the designer need to compare more than 1 trillion lotteries. But by our equivalence result, it is enough to compare 15 lotteries. Another important consequence is that even if we weaken sd-strategy-proofness to a requirement that constrains agents' announcement to a prespecified set, we cannot escape from existing impossibility results such as the Gibbard random dictatorship theorem (Gibbard, 1977).

Our equivalence result generalizes Sato (2013a). Sato (2013a) restricts attention to deterministic rules and considers two domain conditions: "connectedness" and the "non-restoration property". Given two preference relations over objects, we can always change one to the other by consecutively switching two adjacent objects. Two preference relations are connected in a domain if we can change one to the other by performing such "adjacent-pair-switch" operations without leaving the domain. A domain is connected if any two preference relations are connected in the domain. The non-restoration property says that for each pair of connected preference relations, we can change one to the other by performing the adjacent-pair-switch operations, without leaving the domain and without reversing the rankings of any two objects twice. By Sato (2013a), for deterministic rules, if the domain is connected and satisfies the non-restoration property, then e-adjacent strategy-proofness and e-strategy-proofness are equivalent ${ }^{4}$ We find that the non-restoration property, together with connectedness, remains sufficient for the equivalence of sd-adjacent strategy-proofness and sd-strategy-proofness. This is not covered by Carroll (2012) who shows the same equivalence for the "polyhedral type space". On the other hand, for the $d l$ and $u l$-extensions, the non-restoration property is not sufficient (Example 3). We identify domain conditions that guarantee the equivalence for the $d l$ - and $u l$-extensions, too. The universal domain satisfies all these conditions.

The sufficiency of e-adjacent strategy-proofness has two important corollaries. The first one pertains to another strategic property, which we call lie monotonicity. Lie monotonicity requires that

\footnotetext{
${ }^{4}$ In the deterministic case, extensions play no role, so that there is only one notion of strategy-proofness (and similarly for adjacent strategy-proofness). Also, Sato (2013b) shows that when indifferences among objects are allowed, even for deterministic rules, e-adjacent strategy-proofness and e-strategy-proofness are not equivalent unless each preference relation in the domain has at most one indifference class that contains two objects.
} 
each agent's welfare weakly decrease as he reports increasingly bigger lies. Given two preference relations over objects, one is a bigger lie than the other if the former is obtained from the latter by switching some pairs of adjacent objects, where each such switching is a lie according to the true preference relation. Clearly, lie monotonicity is stronger than strategy-proofness: strategyproofness says that truth-telling is a weakly dominant strategy but it is silent on how the welfare of an agent responds to the degree of lying. We show that for each $e \in\{s d, d l, u l\}$, e-strategyproofness is equivalent to e-lie monotonicity (Theorem 2). Thus, if our objective is to design an e-strategy-proof rule, we should distinguish small lies from big ones by punishing the latter more

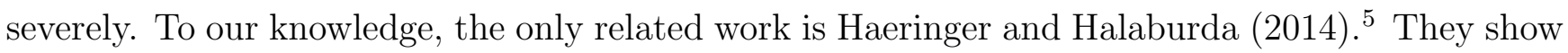
the equivalence of sd-strategy-proofness and sd-lie monotonicity, under a weaker domain condition than the non-restoration property.

The sufficiency of e-adjacent strategy-proofness also reveals an interesting connection among three notions of strategy-proofness. It is clear that sd-strategy-proofness implies dl-strategyproofness and ul-strategy-proofness. Unless there are only three objects, the converse - that $d l$ strategy-proofness and ul-strategy-proofness together imply sd-strategy-proofness - cannot be established simply by checking the definition. However, using the characterization of the three strategy-proofness notions (Remark 2), we show that the converse also holds. That is, sd-strategyproofness is equivalent to the combination of $d l$-strategy-proofness and ul-strategy-proofness (Theorem 3). This "decomposition" result is quite surprising for the following reason. Roughly speaking, by imposing $d l$-strategy-proofness, we only require immunity to misrepresentation by agents whose von Neumann-Morgenstern (vNM) utility functions assign 1 to the most preferred object, $\alpha$ to the second most preferred object, $\alpha^{2}$ to the third most preferred object, and so on, where $\alpha \rightarrow 0^{+}$. Similarly, by imposing ul-strategy-proofness, we only require immunity to misrepresentation by agents whose vNM utility functions assign -1 to the least preferred object, $-\alpha$ to the second least preferred object, $-\alpha^{2}$ to the third least preferred object, and so on, where $\alpha \rightarrow 0^{+}$. Thus, agents with these two extreme types of preferences cannot manipulate a $d l$ - and ul-strategy-proof rule, but we do not know if the same is true for agents with other (vNM or not) preferences. Our decomposition result says that it is sufficient to check for the two extremes.

Concerning efficiency, we study the logical relation among three notions of efficiency: $s d-, d l-$, and $u l$-efficiency. It follows by definition that each of $d l$-and $u l$-efficiency implies sd-efficiency. We show that the converse is also true. That is, $s d$-, $d l$-, and $u l$-efficiency are equivalent (Theorem 4). The preferences obtained by the $d l$-extension (and similarly for the $u l$-extension) are a "completion" of the preferences obtained by the $s d$-extension. Thus, intuitively, it seems feasible to find an assignment that "dl-Pareto dominates" an sd-efficient assignment. However, this turns out to

\footnotetext{
${ }^{5}$ Haeringer and Halaburda (2014) use the term "monotone strategy-proofness".

${ }^{6}$ They also show that for deterministic rules, the equivalence holds without any domain conditions.
} 
be impossible primarily because of a property the $s d$ - and $d l$-extensions share, and hence the equivalence of $s d$ - and dl-efficiency. Abdulkadiroğlu and Sönmez (2003a) and McLennan (2002) provide characterizations of $s d$-efficiency. These characterizations also apply to $d l$-efficiency and ul-efficiency.

We also propose a family of rules that generalize the serial rule (Bogomolnaia and Moulin, 2001). We call them the generalized serial rules. Recall that the serial rule is defined by an algorithm that allows agents to consume (probability) shares of objects over an imaginary timeline. In this algorithm, the consumption speed is the same across all agents and all objects, so that the serial rule satisfies anonymity (the names of agents do not matter) and neutrality (the names of objects do not matter). However, a mechanism designer may seek to "favor" particular preferences, without losing anonymity. For example, in allocation of university housing units, if single rooms are in high demand, the designer may give a priority to students who prefer double rooms to single rooms (thus, neutrality is violated). The serial rule cannot implement such asymmetric treatment because the consumption speed is associated with agents.7

This motivates us to modify the algorithm underlying the serial rule. An (allocation) speed function specifies, for each object and each time $t$, the speed at which shares of that object are allocated at time $t$. Thus, the speed varies across objects and time (but not across agents). Each generalized serial rule is associated with a speed function (see Section 6 for a formal definition). For instance, a speed function may be such that shares of object $a$ are allocated at speed 1 during the time interval $[0,1]$ and at speed 2 during [1,2]; and shares of object $b$ are allocated at speed 2 at any point in time (but for all agents consuming object $b$, the speed is 2 ). The generalized serial rule associated with this speed function favors those who prefer $b$ to $a$ against those who prefer $a$ to $b$ : the former agents can consume object $b$ quickly, and if their consumption of object $b$ is less than 1 , they can move on to the next most preferred objects while other agents are still consuming their most preferred objects.

We assess the generalized serial rules and the random priority rule in terms of efficiency, noenvy, strategy-proofness. No-envy is a fairness property requiring that no agent prefer someone else's lottery to his own. Each extension gives rise to a notion of no-envy, and sd-no-envy implies $d l$-no-envy and ul-no-envy (but even the combination of $d l$-no-envy and ul-no-envy does not imply sd-no-envy). We find that the generalized serial rules are sd-efficient (and hence dl-efficient and ul-efficient) whereas the random priority rule is not (Theorem 5). With regard to no-envy, the generalized serial rules and the random priority rule satisfy $d l$-no-envy (Theorem 6). Although the serial rule satisfies sd-no-envy, some generalized serial rules violate it. Finally, as far as strategy-proofness is concerned, the serial rule is $d l$-strategy-proof and the random priority rule

\footnotetext{
${ }^{7}$ Bogomolnaia and Moulin (2001) consider an algorithm where the consumption speed varies across agents and time, but not across objects. The rule defined by this algorithm violates anonymity and $d l-n o-e n v y$.
} 
is sd-strategy-proof (Theorem 7). If the speed function varies too much across objects (e.g., the allocation speed for one object is too low compared to those for other objects), then the generalized serial rule is not $d l$-strategy-proof (Example 8). Properties of the serial rule and the random priority rule for the $s d$-extension case are already known (Bogomolnaia and Moulin, 2001). However, by allowing for the $d l$ - and $u l$-extensions as well, we discover additional properties of these rules and explain why they violate some properties associated with the $s d$-extension. Also, our results yield the by-product that Bogomolnaia and Moulin's (2001) impossibility result - namely that no rule satisfies sd-efficiency, equal treatment of equals 8 , and sd-strategy-proofness-hinges critically on the extension chosen?

As is true of any economic model, many results in probabilistic assignment rest on assumptions the modeller makes, and the connection between the two reveals itself most clearly when different assumptions are imposed. In light of this, it is best to interpret our extensions as a tool for understanding the role the traditional assumption (the $s d$-extension) plays in the ordinal approach. This "extension approach" allows us to study probabilistic assignment under various assumptions (extensions) and provide a new perspective on existing results.

The rest of the paper proceeds as follows. We discuss related literature in Section 2 and set up the model in Section 3. We present results on strategy-proofness in Section 4 and results on efficiency and no-envy in Section 5. We introduce the generalized serial rules and study their properties, together with the random priority rule, in Section 6. We conclude in Section 7. Omitted proofs are in Appendices $\mathrm{A}$ and $\mathrm{B}$.

\section{$2 \quad$ Related Literature}

Our results on strategy-proofness, adjacent strategy-proofness, and lie monotonicity are not specific to probabilistic assignment; they apply to any model - e.g., voting, school choice, and house allocation with existing tenants - where agents report preferences over a finite set of sure outcomes and receive lotteries defined over them. As discussed in detail in the introduction, Carroll (2012), Sato (2013a), and Haeringer and Halaburda (2014) are closely related papers.

The first model of probabilistic assignment is due to Hylland and Zeckhauser (1979). They assume that agents have vNM preferences and propose a rule that first entitles each agent to a budget and then lets them trade probability shares of objects in a pseudo-market mechanism. This rule satisfies ex ante efficiency and no-envy, but not strategy-proofness. In fact, no rule meets the three requirements in this model (Zhou, 1990).

\footnotetext{
${ }^{8}$ Equal treatment of equals requires that agents with the same preference relation receive the same lottery up to indifference.

${ }^{9}$ Kasajima (2013) shows that even on the single-peaked preference domain, the three properties are incompatible.
} 
In contrast with this cardinal approach, recent papers adopt the ordinal framework in which agents only submit preferences over objects. To define properties of assignment rules based on the ordinal information, Bogomolnaia and Moulin (2001) extend preferences over objects to preferences over lotteries by the $s d$-extension. They then introduce sd-efficiency 10 , a concept that is intermediate in strength between ex post and ex ante efficiency. They also propose the serial rule and study its properties, together with the random priority rule. Subsequent works generalize their model in several directions to allow for the following possibilities: (i) there are multiple copies of each object; (ii) agents may choose not to receive any object, that is, to receive a "null" object; (iii) agents may receive more than one object; (iv) agents may be indifferent among some objects; and (v) agents privately own fractions of objects. The following papers explore these variations in different combinations: Bogomolnaia and Heo (2012), Che and Kojima (2010), Hashimoto et al. (2014), Katta and Sethuraman (2006), Kesten (2009), Kojima (2009), Liu and Pycia (2012), and Yilmaz $(2009,2010)$. These papers too take the ordinal approach based on the $s d$-extension. In particular, Bogomolnaia and Heo (2012) and Hashimoto et al. (2014) characterize the serial rule by sd-efficiency, sd-no-envy, and some invariance conditions.

Several concurrent papers take the ordinal approach based on the $d l$-extension. Schulman and Vazirani (2012) study the problem of allocating perfectly divisible commodities and propose the "synchronized greedy" rule, which generalizes the serial rule to the case where agents do not necessarily have integer-valued demand. If object supplies and agent demands satisfy a certain condition, the synchronized greedy rule inherits $d l$-efficiency, sd-no-envy, and dl-strategy-proofness from the serial rule. Saban and Sethuraman (2013) identify a sufficient and necessary condition on the number of agents and the supply of commodities under which there is a rule satisfying $d l$-efficiency, $d l$-no-envy, and $d l$-strategy-proofness. They also show that in the unit demand case, which we consider, the serial rule is not the only rule with the latter three properties. Bogomolnaia (2012) shows that the serial rule lexicographically maximizes the profile of probabilities for preferred objects. On the other hand, the following papers consider the $d l$-extension in other models: Aziz, Brandl, and Brandt (2014, Arrovian voting model), Alcalde and Silva-Reus (2013, object allocation problems with priorities), Alcalde (2013, house allocation problems with existing tenants).

The notion of sd-efficiency has become a topic of independent interest. Abdulkadiroğlu and Sönmez (2003a) characterize sd-efficiency by a dominance concept defined over sets of assignments. McLennan (2002) proves a welfare theorem involving sd-efficiency, using a separating hyperplane theorem for polyhedra.11 Katta and Sethuraman (2006) study sd-efficiency and generalize the

\footnotetext{
${ }^{10}$ Bogomolnaia and Moulin (2001) call it ordinal efficiency.

${ }^{11}$ Manea (2008) provides an alternative constructive proof and Carroll (2010) extends McLennan (2002) to a more general type space.
} 
serial rule in a more general setup that permits indifferences among objects. Liu and Pycia (2012) show that if a sequence of rules consists of uniform randomizations over efficient deterministic rules, then the sequence is asymptotically sd-efficient. Because $s d$-efficiency, dl-efficiency, and ul-efficiency are equivalent, these papers can also be viewed as providing additional properties of the common efficiency notion.

\section{The Model}

Let $\boldsymbol{A} \equiv\{1, \cdots, n\}$ be the set of objects and $\boldsymbol{N} \equiv\{1, \cdots, n\}$ the set of agents. Assume that $n \geq 2$, and note that we have the same number of agents and objects. We denote objects by $k, \ell, k^{\prime}, \ell^{\prime}$, and so on, and agents by $i, j, i^{\prime}, j^{\prime}$, and so on. Let $\mathcal{R}(\boldsymbol{A})$ be the set of all complete, transitive, and anti-symmetric preference relations over $A$. For each $i \in N$, let $\boldsymbol{R}_{\boldsymbol{i}} \in \mathcal{R}(A)$ be agent $i$ 's preference relation over $A$. Let $\boldsymbol{P}_{\boldsymbol{i}}$ and $\boldsymbol{I}_{\boldsymbol{i}}$ be the strict preference and indifference relations, respectively, associated with $R_{i}$. Also, for each $k \in\{1, \cdots, n\}$, let $\boldsymbol{k}\left(\boldsymbol{R}_{\boldsymbol{i}}\right)$ be the object ranked $k$ th according to $R_{i}$. An economy is a profile $\boldsymbol{R} \equiv\left(R_{i}\right)_{i \in N}$. Let $\mathcal{R}(\boldsymbol{A})^{\boldsymbol{N}}$ denote the set of all economies.

Let $\boldsymbol{\Delta} \boldsymbol{A}$ be the set of all lotteries over $A$. Given an economy $R \in \mathcal{R}(A)^{N}$, a (feasible) assignment for $R$ is a profile $\boldsymbol{\pi} \equiv\left(\pi_{i}\right)_{i \in N}$ such that (i) for each $i \in N, \pi_{i} \in \Delta A$; and (ii) for each $k \in A, \sum_{i \in N} \pi_{i k}=1$. We call $\pi_{i}$ agent $i$ 's lottery. Let $\Pi$ be the set of all assignments. If, for each $i \in N, \pi_{i}$ is a degenerate lottery, then $\pi$ is a deterministic assignment. By the Birkhoffvon Neumann theorem (Birkhoff, 1946; von Neumann, 1953), each probabilistic assignment can be written as a convex combination of deterministic assignments ${ }^{12}$ An (assignment) rule is a mapping $\varphi: \mathcal{R}(A)^{N} \rightarrow \Pi$. Two rules have been studied extensively in the literature: the serial and random priority rules. In Section 6, we define these rules and propose a family of rules that generalize the serial rule.

Our definition of a rule takes preferences over objects as an input. This, however, does not mean that agents have no preferences over lotteries. They have preferences over lotteries, e.g., vNM preferences, but the rule only allows them to submit preferences over objects. Such "ordinal" rules have the advantage that they are simple to implement. Collecting information on preferences over lotteries presents a number of practical issues, and most real-life allocation problems that use lotteries only elicit preferences over objects (e.g., on-campus housing allocation in colleges and student placement in public schools).

However, in order to speak of properties of assignments and rules, we should first specify how agents evaluate lotteries. A standard approach in the literature is to apply (first-order)

\footnotetext{
${ }^{12}$ Budish et al. (2013, Theorem 1) and Kojima and Manea (2010, Proposition 1) provide a generalization of this result.
} 
stochastic dominance to preferences over objects and obtain preferences over lotteries (Gibbard, 1977; Bogomolnaia and Moulin, 2001). We call this procedure the "sd-extension". The preferences obtained by the $s d$-extension are very incomplete: a large number of lotteries cannot be compared. However, they are the most we can infer about preferences over lotteries because agents only report ordinal preferences over objects. Our objective is to better understand the current practice with the aid of alternatives to the $s d$-extension.

Let $\mathcal{R}(\Delta \boldsymbol{A})$ be the set of all preferences over $\Delta A$. An extension is a mapping $e: \mathcal{R}(A) \rightarrow$ $\mathcal{R}(\Delta A)$ such that for each for each $R_{0} \in \mathcal{R}(A)$, the restriction of $e\left(R_{0}\right)$ to $A$ coincides with $R_{0}{ }^{13}{ }^{14}$ For each $R_{0} \in \mathcal{R}(A)$, let $\boldsymbol{R}_{\mathbf{0}}^{\boldsymbol{e}} \equiv e\left(R_{0}\right)$. The strict preference and indifference relations associated with $R_{0}^{e}$ are denoted by $\boldsymbol{P}_{\mathbf{0}}^{\boldsymbol{e}}$ and $\boldsymbol{I}_{\mathbf{0}}^{\boldsymbol{e}}$, respectively. Clearly, the sd-extension satisfies our definition of an extension.

We consider two alternatives to the sd-extension, which give lexicographic preferences over lotteries (Hausner, 1954; Chipman, 1960). The first alternative is the downward lexicographic extension, or simply the $\boldsymbol{d} \boldsymbol{l}$-extension. The preferences obtained by the $d \boldsymbol{l}$-extension are as follows. Given two lotteries, the lottery that assigns a higher probability to the most preferred object is preferred; if the two lotteries assign equal probability, then the lottery that assigns a higher probability to the second most preferred object is preferred; if the two lotteries assign equal probability again, then the probabilities for the third most preferred object are compared, and so on. Formally, for each $R_{0} \in \mathcal{R}(A)$ and each pair $\pi_{0}, \pi_{0}^{\prime} \in \Delta A, \pi_{0} R_{0}^{d l} \pi_{0}^{\prime}$ if either (i) there is $k \in\{1, \cdots, n\}$ such that for each $h \leq k-1, \pi_{0 h\left(R_{0}\right)}=\pi_{0 h\left(R_{0}\right)}^{\prime}$ and $\pi_{0 k\left(R_{0}\right)}>\pi_{0 k\left(R_{0}\right)}^{\prime}$; or (ii) $\pi=\pi^{\prime}$.

The second extension also performs lexicographic comparison, but in the opposite direction. The upward lexicographic dominance extension, or the $\boldsymbol{u l}$-extension, gives the following preferences. Given two lotteries, the lottery that assigns a lower probability to the least preferred object is preferred; if the two lotteries assign equal probability, then the lottery that assigns a lower probability to the second least preferred object is preferred; and so on. Formally, for each $R_{0} \in \mathcal{R}(A)$ and each pair $\pi_{0}, \pi_{0}^{\prime} \in \Delta A, \pi_{0} R_{0}^{u l} \pi_{0}^{\prime}$ if either (i) there is $k \in\{1, \cdots, m\}$ such that for each $h \geq k+1, \pi_{0 h\left(R_{0}\right)}=\pi_{0 h\left(R_{0}\right)}^{\prime}$ and $\pi_{0 k\left(R_{0}\right)}<\pi_{0 k\left(R_{0}\right)}^{\prime}$; or (ii) $\pi_{0}=\pi_{0}^{\prime}$.

As is transparent from the definitions, the $d l$ - and $u l$-extensions are similar but at the same time, diametrically opposite to each other. They are similar in that lexicographic comparison is used; they are opposite in that one first maximizes probabilities for preferred objects whereas the other first minimizes probabilities for less preferred objects. This observation can be formally stated using the notion of "duality" 15 Also, in contrast with the $s d$-extension, the $d l$ - and $u l$-extensions

\footnotetext{
${ }^{13}$ Preference relations and lotteries that are not associated with any particular agent have the subscript "0".

${ }^{14}$ An alternative way of presenting the same idea is to say that agents' preferences over lotteries are drawn from some set of admissible preferences (e.g., the set of all preferences satisfying monotonicity if the $s d$-extension is considered) and a rule only allows them to submit ordinal preferences over objects.

${ }^{15}$ Let $X$ be an arbitrary set. For each binary relation $B$ over $X$, let $\boldsymbol{B}^{-\mathbf{1}}$ be the inverse of $B$; i.e., for each pair
} 
give complete (in fact, linear) preferences over lotteries. While most of the work taking the ordinal approach consider the $s d$-extension, some recent papers adopt the $d l$-extension: Schulman and Vazirani (2012), Bogomolnaia (2012), Saban and Sethuraman (2013), Aziz, Brandl, and Brandt (2014), Alcalde and Silva-Reus (2013), and Alcalde (2013).

Let $e$ and $\hat{e}$ be extensions. Then $\boldsymbol{e}$ is contained in $\hat{\boldsymbol{e}}$, denoted $\boldsymbol{e} \subseteq \hat{\boldsymbol{e}}$, if for each $R_{0} \in \mathcal{R}(A)$ and each pair $\pi_{0}, \pi_{0}^{\prime} \in \Delta A, \pi_{0} R_{0}^{e} \pi_{0}^{\prime}$ implies $\pi_{0} R_{0}^{\hat{e}} \pi_{0}^{\prime}$. The relations $e \subsetneq \hat{e}$ and $e=\hat{e}$ are defined in the standard way. Note that $s d \subsetneq d l, s d \subsetneq u l, d l \nsubseteq u l$, and $u l \nsubseteq d l$.

In what follows, we consider several properties, or axioms, of rules. The content of these properties varies depending on the extension chosen. Therefore, we state them using a general extension $e$.

\section{Strategy-proofness}

Our first axiom concerns the strategic behavior of agents. Below we discuss properties of the set from which agents' preferences are drawn. Therefore, instead of working with the universal domain $\mathcal{R}(A)$, we consider an arbitrary subset of $\mathcal{R}(A)$. A (preference) domain is a non-empty set $\mathcal{D} \subseteq \mathcal{R}(A)$. Given domain $\mathcal{D}$, a rule is a mapping $\varphi: \mathcal{D}^{N} \rightarrow \Pi$. When preferences are private information, agents may find it in their interest to misrepresent their preferences. The following property requires that whatever other agents' announcements are, no agent ever profit from lying about his preferences.

e-Strategy-proofness: For each $R \in \mathcal{D}^{N}$, each $i \in N$, and each $R_{i}^{\prime} \in \mathcal{D}, \varphi_{i}(R) R_{i}^{e} \varphi_{i}\left(R_{i}^{\prime}, R_{-i}\right)$.

The inclusion relation among extensions yields a logical relation among different notions of strategy-proofness. Let $e$ and $\hat{e}$ be extensions such that $e \subseteq \hat{e}$. Then by definition, e-strategyproofness implies $\hat{e}$-strategy-proofness. Since $s d \subsetneq d l$ and $s d \subsetneq u l$, sd-strategy-proofness implies $d l$ and ul-strategy-proofness.

One way to weaken e-strategy-proofness is to constrain the set of possible lies an agent can choose from. By definition, e-strategy-proofness allows each agent to announce any preference relation in the domain. However, there are situations in which agents are constrained to choose a preference relation that is somewhat close to the truth. This may be because "big" lies are not so credible or because agents can lie only about the part of private information that has not been disclosed yet (see Sato (2013a) and Carroll (2012) for a detailed discussion). Therefore, it is

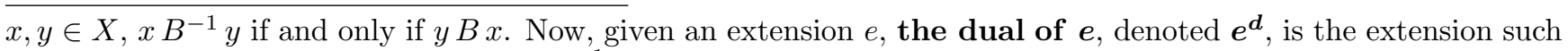
that for each $R_{0} \in \mathcal{R}(A), R_{0}^{e^{d}}=\left(\left(R_{0}^{-1}\right)^{e}\right)^{-1}$ (or equivalently, $\left.\left(R^{e^{d}}\right)^{-1}=\left(R^{-1}\right)^{e}\right)$. An extension is self-dual if it is the dual of itself. The $d l$ - and $u l$-extensions are dual; and the $s d$-extension is self-dual. 


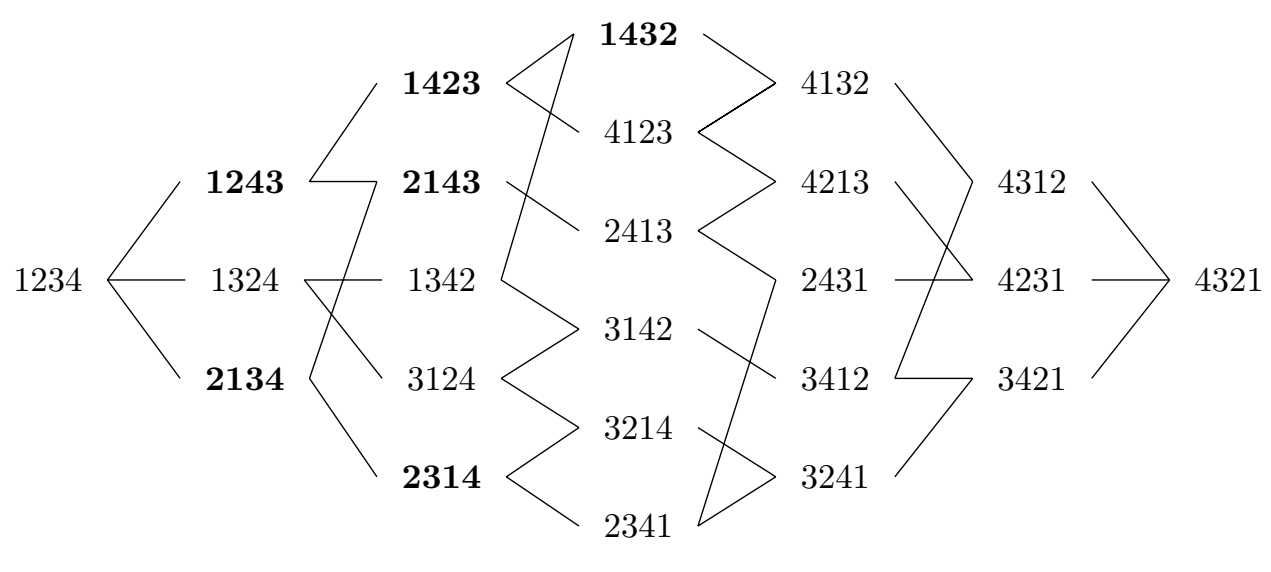

Figure 2: Metrizing the space of preferences (Example 1). Let $A \equiv\{1,2,3,4\}$. In the figure, "1432", for instance, stands for the preference relation $R_{0} \in \mathcal{R}(A)$ such that $1 P_{0} 4 P_{0} 3 P_{0} 2$. Adjacent preference relations are connected by an arc. If $\mathcal{D}=\mathcal{R}(A)$, the distance between two preference relations is the smallest number of arcs we go through when traveling from one preference relation to the other. For example, $d(1432,2314)=5$.

interesting to consider a strategic property that requires a weaker form of immunity to misrepresentation, where misrepresentation is restricted to some prespecified set.

To formalize this idea, we follow Sato's (2013) approach and metrize the preference domain as follows. First, for each pair $R_{0}, R_{0}^{\prime} \in \mathcal{D}, \boldsymbol{R}_{\mathbf{0}}^{\prime}$ is adjacent to $\boldsymbol{R}_{\mathbf{0}}$ if $R_{0}^{\prime}$ is obtained from $R_{0}$ by switching two objects whose rankings according to $R_{0}$ are adjacent; i.e., there is $\hat{k} \in\{1, \cdots, n\}$ such that (i) $\hat{k}\left(R_{0}\right)=(\hat{k}+1)\left(R_{0}^{\prime}\right)$; (ii) $(\hat{k}+1)\left(R_{0}\right)=\hat{k}\left(R_{0}^{\prime}\right)$; and (iii) for each $k \in\{1, \cdots, n\} \backslash\{\hat{k}, \hat{k}+1\}$, $k\left(R_{0}\right)=k\left(R_{0}^{\prime}\right)$. For each pair $R_{0}, R_{0}^{\prime} \in \mathcal{D}$, a path from $\boldsymbol{R}_{\mathbf{0}}$ to $\boldsymbol{R}_{\mathbf{0}}^{\prime}$ in $\mathcal{D}$ is a sequence of preference relations $\left\{R_{0}^{0}, R_{0}^{1}, \cdots, R_{0}^{h}\right\}$ in $\mathcal{D}$ such that (i) $R_{0}^{0}=R_{0}$ and $R_{0}^{h}=R_{0}^{\prime}$; and (ii) for each $h^{\prime} \in\{0, \cdots, h-1\}, R_{0}^{h^{\prime}}$ and $R_{0}^{h^{\prime}+1}$ are adjacent. We call $h$ the length of the path. Next, define a metric $d(\cdot, \cdot)$ on $\mathcal{D}$ : for each pair $R_{0}, R_{0}^{\prime} \in \mathcal{D}$, if there is a path from $R_{0}$ to $R_{0}^{\prime}$ in $\mathcal{D}$, let $\boldsymbol{d}\left(\boldsymbol{R}_{\mathbf{0}}, \boldsymbol{R}_{\mathbf{0}}^{\prime}\right)$ be the length of a shortest path from $R_{0}$ to $R_{0}^{\prime}$ in $\mathcal{D}$; otherwise, $d\left(R_{0}, R_{0}^{\prime}\right)=\infty$. If, for example, $\mathcal{D}=\mathcal{R}(A), d(\cdot, \cdot)$ coincides with the Kemeny metric (Kemeny, 1959; Kemeny and Snell, 1962), but in general, the two are not the same 16

Throughout this section, we write, for instance, "1432" for the preference relation $R_{0}$ such that $1 P_{0} 4 P_{0} 3 P_{0} 2$.

Example 1. Metrizing the space of preferences when there are four objects. Let $A \equiv\{1,2,3,4\}$ and $\mathcal{D} \equiv \mathcal{R}(A)$. Refer to Figure 2. Since there are four objects, each preference relation has three adjacent ones, each of which is connected by an arc. The distance between preference relations 1432 and

\footnotetext{
${ }^{16}$ Whenever $\mathcal{D}$ is a connected domain satisfying the non-restoration property, $d(\cdot, \cdot)$ coincides with the Kemeny metric. Also, while we mechanically define the metric $d(\cdot, \cdot)$, it can be derived as a consequence of a list of axioms on metrics over $\mathcal{R}(A)$ (Kemeny, 1959).
} 
2314, say, is the smallest number of arcs we go through when traveling from 1432 to 2314 . There are multiple paths achieving that smallest number, and the path $\{1432,1423,1243,2143,2134,2314\}$ (boldfaced) is one of them. Therefore, $d(1432,2314)=5$.

With the metric $d(\cdot, \cdot)$ in mind, e-strategy-proofness can be viewed as permitting the possibility that each agent can submit any preference relation in the domain, regardless of how far it is from his true preference relation according to $d(\cdot, \cdot)$. As an extreme weakening of e-strategy-proofness, we assume that each agent can only announce a preference relation that is closest to his true preference relation, and require that no agent ever benefit from such manipulation.

e-Adjacent strategy-proofness: For each $R \in \mathcal{D}^{N}$, each $i \in N$, and each $R_{i}^{\prime} \in \mathcal{D}$ such that $R_{i}^{\prime}$ is adjacent to $R_{i}, \varphi_{i}(R) R_{i}^{e} \varphi_{i}\left(R_{i}^{\prime}, R_{-i}\right)$.

In general, e-adjacent strategy-proofness is weaker than e-strategy-proofness. We ask under what conditions on the domain the two are equivalent. A simple necessary condition is that each pair of preference relations in the domain should be "connected" by a path. However, this is not sufficient; the domain should have more structure. Our sufficient conditions vary depending on the extension under consideration. Now we introduce them.

Two preference relations $R_{0}, R_{0}^{\prime} \in \mathcal{D}$ are connected in $\mathcal{D}$ if there is a path from $R_{0}$ to $R_{0}^{\prime}$ in $\mathcal{D}$. The domain $\mathcal{D}$ is connected if each pair of preference relations in $\mathcal{D}$ are connected in $\mathcal{D}$. Let $R_{0}, R_{0}^{\prime} \in \mathcal{D}$, and let $\left\{R_{0}^{0}, R_{0}^{1}, \cdots, R_{0}^{h}\right\}$ be a path from $R_{0}$ to $R_{0}^{\prime}$ in $\mathcal{D}$. For each pair $k, \ell \in A$, the path is with $\{\boldsymbol{k}, \boldsymbol{\ell}\}$-restoration if for some $h_{1}, h_{2}, h_{3} \in\{0,1, \cdots, h\}, k P_{0}^{h_{1}} \ell, \ell P_{0}^{h_{2}} k$, and $k P_{0}^{h_{3}} \ell$. The path is without restoration if for any pair $k, \ell \in A$, the path is not with $\{k, \ell\}$-restoration. The domain $\mathcal{D}$ satisfies the non-restoration property if for each pair of connected preference relations $R_{0}, R_{0}^{\prime} \in \mathcal{D}$, there is a path from $R_{0}$ to $R_{0}^{\prime}$ in $\mathcal{D}$ without restoration (Sato, 2013a).

Connectedness and the non-restoration property are sufficient for the equivalence of sd-adjacent strategy-proofness and sd-strategy-proofness. However, they are not if the $d l$ - and ul-extensions are considered. To define stronger conditions, let $R_{0}, R_{0}^{\prime} \in \mathcal{D}$. Let $\boldsymbol{k}^{*}\left(\boldsymbol{R}_{\mathbf{0}} \mid \boldsymbol{R}_{\mathbf{0}}^{\prime}\right) \equiv(\min \{1 \leq k \leq$ $\left.\left.n: k\left(R_{0}\right) \neq k\left(R_{0}^{\prime}\right)\right\}\right)\left(R_{0}\right)$ be the object that $R_{0}$ ranks highest among those whose rankings differ according to $R_{0}$ and $R_{0}^{\prime}$. A path $\left\{R_{0}^{0}, R_{0}^{1}, \cdots, R_{0}^{h}\right\}$ from $R_{0}$ to $R_{0}^{\prime}$ in $\mathcal{D}$ moves preferred objects first if for each $\hat{h} \in\{0, \cdots, h-1\}$, the ranking of object $k^{*}\left(R_{0}^{\prime} \mid R_{0}^{\hat{h}}\right)$ is higher according to $R_{0}^{\hat{h}+1}$ than according to $R_{0}^{\hat{h}}$. Now the domain $\mathcal{D}$ satisfies the preferred-objects-first (POF) path property if for each pair of connected preference relations $R_{0}, R_{0}^{\prime} \in \mathcal{D}$, there is a path from $R_{0}$ to $R_{0}^{\prime}$ in $\mathcal{D}$ moving preferred objects first.

Similarly, let $R_{0}, R_{0}^{\prime} \in \mathcal{D}$. Let $\boldsymbol{k}_{*}\left(\boldsymbol{R}_{\mathbf{0}} \mid \boldsymbol{R}_{\mathbf{0}}^{\prime}\right) \equiv\left(\max \left\{1 \leq k \leq n: k\left(R_{0}\right) \neq k\left(R_{0}^{\prime}\right)\right\}\right)\left(R_{0}\right)$ be the object that $R_{0}$ ranks lowest among those whose rankings differ according to $R_{0}$ and $R_{0}^{\prime}$. A path $\left\{R_{0}^{0}, R_{0}^{1}, \cdots, R_{0}^{h}\right\}$ from $R_{0}$ to $R_{0}^{\prime}$ in $\mathcal{D}$ moves less preferred objects first if for each $\hat{h} \in$ 
$\{0, \cdots, h-1\}$, the ranking of object $k_{*}\left(R_{0}^{\prime} \mid R_{0}^{\hat{h}}\right)$ is lower according to $R_{0}^{\hat{h}+1}$ than according to $R_{0}^{\hat{h}}$. Now $\mathcal{D}$ satisfies the less-preferred-objects-first (LOF) path property if for each pair of connected preference relations $R_{0}, R_{0}^{\prime} \in \mathcal{D}$, there is a path from $R_{0}$ to $R_{0}^{\prime}$ in $\mathcal{D}$ moving less preferred objects first.

Each path from one preference relation to another moving preferred or less preferred objects first is without restoration. Thus, the POF and LOF path properties each imply the non-restoration property, but neither of the converses holds. The universal domain, $\mathcal{R}(A)$, satisfies all of the three domain properties.

Example 2. Illustrating domain properties. Let $A \equiv\{1,2,3\}, \mathcal{D} \equiv\{123,213,231,321\}$, and $\hat{\mathcal{D}} \equiv\{123,132,312,321\}$.

Non-restoration property: Consider the path $\{123,213,231,321\}$ from 123 to 321 in $\mathcal{D}$. The path is without restoration. It is simple to check that for each pair of preference relations in $\mathcal{D}$, there is a path without restoration. Thus, $\mathcal{D}$ satisfies the non-restoration property; similarly, so does $\hat{\mathcal{D}}$.

POF path property: First, note that $k^{*}(321 \mid 123)=3$. The path from 123 to 321 in $\mathcal{D}$ that moves preferred objects first should move object 3 first. There is no such path in $\mathcal{D}$, so that $\mathcal{D}$ does not satisfy the POF path property. However, the path $\{123,132,312,321\}$ from 123 to 321 in $\hat{\mathcal{D}}$ moves preferred objects first. Each pair of preference relations in $\hat{\mathcal{D}}$ has a path moving preferred objects first. Thus, $\hat{\mathcal{D}}$ satisfies the POF path property.

LOF path property: Since $k_{*}(321 \mid 123)=1$, the path from 123 to 321 in $\mathcal{D}$ that moves less preferred objects first should move object 1 first. The path $\{123,213,231,321\}$ in $\mathcal{D}$ moves less preferred objects first, and each pair of preference relations in $\mathcal{D}$ has such path. Thus, $\mathcal{D}$ satisfies the LOF path property; however, $\hat{\mathcal{D}}$ does not.

While there are some contexts where e-adjacent strategy-proofness is compelling (Sato, 2013a), our interest mainly concerns the convenience it provides in checking the stronger axiom, e-strategyproofness. As Theorem 1 states below, under various assumptions on the domain, for each $e \in$ $\{s d, d l, u l\}$, e-adjacent strategy-proofness implies e-strategy-proofness. Thus, the task of verifying the latter property can be simplified very much. The proof is in Appendix A.

Theorem 1. Let $\mathcal{D}$ be a connected domain.

(1) If $\mathcal{D}$ satisfies the non-restoration property, then sd-adjacent strategy-proofness is equivalent to $s d$-strategy-proofness.

(2) If $\mathcal{D}$ satisfies the POF path property, then dl-adjacent strategy-proofness is equivalent to $d l$-strategy-proofness. 
(3) If $\mathcal{D}$ satisfies the LOF path property, then ul-adjacent strategy-proofness is equivalent to ul-strategy-proofness.

Remark 1. When restricted to deterministic rules, for each $e \in\{s d, d l, u l\}$, e-(adjacent) strategyproofness reduces to the same requirement. Sato (2013a) shows that for deterministic rules, the non-restoration property guarantees the equivalence of $e$-adjacent strategy-proofness and e-strategyproofness. Theorem 1 generalizes this result to probabilistic rules. The non-restoration property is still sufficient for the $s d$-extension, but not for the $d l$ - and $u l$-extensions (Example 3 below provides counter-examples) ${ }^{17}$

In several economic environments, Carroll (2012) also identifies conditions on preference domains for the sufficiency to hold. Among others, he shows that if a domain satisfies a certain regularity condition, then sd-adjacent strategy-proofness is equivalent to sd-strategy-proofness. There is no logical relation between his condition and the non-restoration property.

Remark 2. Theorem 1 yields a corollary on the behavior of $s d$-, $d l$-, and ul-strategy-proof rules. Let $\varphi$ be a rule defined on a domain $\mathcal{D}$ satisfying the respective properties in Theorem 1 . In the statement below, we take arbitrary $R \in \mathcal{D}^{N}, i \in N$, and $R_{i}^{\prime} \in \mathcal{D}$ such that $R_{i}^{\prime}$ is adjacent to $R_{i}$. To simplify notation, however, once such $\left(R, i, R_{i}^{\prime}\right)$ is chosen, (i) relabel objects so that $1 P_{i} 2 P_{i} \cdots P_{i} n$; (ii) let $k \in A$ be the object such that $(k+1) P_{i}^{\prime} k$; and (iii) let $\pi \equiv \varphi(R)$ and $\pi^{\prime} \equiv \varphi\left(R_{i}^{\prime}, R_{-i}\right)$.

(i) $\varphi$ is sd-strategy-proof

if and only if for each $R \in \mathcal{D}^{N}$, each $i \in N$, and each $R_{i}^{\prime} \in \mathcal{D}$ adjacent to $R_{i}$, either $(\mathrm{a}) \pi_{i}=\pi_{i}^{\prime}$;

or $\quad(\mathrm{b}) \pi_{i k}>\pi_{i k}^{\prime}, \pi_{i, k+1}<\pi_{i, k+1}^{\prime}$, and for each $\ell \in A \backslash\{k, k+1\}, \pi_{i \ell}=\pi_{i \ell}^{\prime}$.

(ii) $\varphi$ is dl-strategy-proof

if and only if for each $R \in \mathcal{D}^{N}$, each $i \in N$, and each $R_{i}^{\prime} \in \mathcal{D}$ adjacent to $R_{i}$, either (a) $\pi_{i}=\pi_{i}^{\prime}$;

or $\quad$ (b) $\pi_{i k}>\pi_{i k}^{\prime}, \pi_{i, k+1}<\pi_{i, k+1}^{\prime}$, and for each $\ell \in\{1, \cdots, k-1\}, \pi_{i \ell}=\pi_{i \ell}^{\prime}$.

(iii) $\varphi$ is ul-strategy-proof

if and only if for each $R \in \mathcal{D}^{N}$, each $i \in N$, and each $R_{i}^{\prime} \in \mathcal{D}$ adjacent to $R_{i}$, either (a) $\pi_{i}=\pi_{i}^{\prime}$;

or $\quad(\mathrm{b}) \pi_{i k}>\pi_{i k}^{\prime}, \pi_{i, k+1}<\pi_{i, k+1}^{\prime}$, and for each $\ell \in\{k+2, \cdots, n\}, \pi_{i \ell}=\pi_{i \ell}^{\prime}$.

\footnotetext{
${ }^{17}$ In our model, we only consider "strict" preference relations (no two alternatives are indifferent). Sato (2013b) finds that when indifferences are allowed, adjacent strategy-proofness is not sufficient for strategy-proofness even for deterministic rules.
} 
In Appendix A, to prove Theorem 1, we introduce an auxiliary axiom, "e-within- $m$ strategyproofness" $(m \in \mathbb{N})$, which requires that no agent ever gain by reporting a preference relation whose distance from the truth according to $d(\cdot, \cdot)$ is at most $m$. Then we show that for each $m \in \mathbb{N}$, e-within-m strategy-proofness implies e-within- $(m+1)$ strategy-proofness.

The following example shows that for each $e \in\{d l, u l\}$, the non-restoration property is not enough for the equivalence of e-adjacent strategy-proofness and e-strategy-proofness.

Example 3. Insufficiency of the non-restoration property for the $d l$-and ul-extensions. Let $N \equiv$ $\{1,2,3\}, A \equiv\{1,2,3\}$, and $\mathcal{D} \equiv\{123,213,231,321\}$. Note that $\mathcal{D}$ is connected, and satisfies the non-restoration property but not the POF path property. Define a rule $\varphi$ as follows. For each $R_{-1} \in \mathcal{D}^{N \backslash\{1\}}$, let $\varphi_{1}\left(123, R_{-1}\right)=(0.3,0.4,0.3) ; \varphi_{1}\left(213, R_{-1}\right)=(0.2,0.5,0.3) ; \varphi_{1}\left(231, R_{-1}\right)=$ $(0.1,0.5,0.4)$; and $\varphi_{1}\left(321, R_{-1}\right)=(0.4,0.1,0.5)$. Also, for each $R \in \mathcal{D}^{N}, \varphi_{2}(R)=\varphi_{3}(R)=$ $\frac{1}{2}\left[(1,1,1)-\varphi_{1}(R)\right]$. Then $\varphi$ is dl-adjacent strategy-proof. However, since for any $R_{-1} \in \mathcal{D}^{N \backslash\{1\}}$, $\varphi_{1}\left(321, R_{-1}\right)(123)^{d l} \varphi_{1}\left(123, R_{-1}\right), \varphi$ is not $d l$-strategy-proof.

Next, let $\hat{\mathcal{D}} \equiv\{123,132,312,321\}$. Then $\hat{\mathcal{D}}$ is connected, and satisfies the non-restoration property but not the LOF path property. Define a rule $\hat{\varphi}$ that is similar to $\varphi$ except for the following. For each $R_{-1} \in \mathcal{D}^{N \backslash\{1\}}$, let $\hat{\varphi}_{1}\left(123, R_{-1}\right)=(0.3,0.4,0.3) ; \hat{\varphi}_{1}\left(132, R_{-1}\right)=(0.3,0.2,0.5)$; $\hat{\varphi}_{1}\left(312, R_{-1}\right)=(0.2,0.2,0.6)$; and $\hat{\varphi}_{1}\left(321, R_{-1}\right)=(0.1,0.9,0)$. Then $\hat{\varphi}$ is ul-adjacent strategy-proof. However, since for any $R_{-1} \in \mathcal{D}^{N \backslash\{1\}}, \hat{\varphi}_{1}\left(321, R_{-1}\right)(123)^{u l} \hat{\varphi}_{1}\left(123, R_{-1}\right), \hat{\varphi}$ is not ul-strategy-proof.

Next, we introduce an incentive property that strengthens strategy-proofness. Let $R \in \mathcal{D}^{N}$ and $i \in N$. We ask how agent $i$ 's welfare, as measured by $R_{i}^{e}$, is affected as he reports increasingly bigger lies. If a rule is e-strategy-proof, his welfare is maximized when he reports $R_{i}$. We do not know if he is better off with a smaller lie than with a larger lie. Our result below says that an e-strategy-proof rule should respond monotonically to the degree of lying.

To state this formally, let $R_{0} \in \mathcal{D}$. Define an order $\geq_{\boldsymbol{R}_{\mathbf{0}}}$ over $\mathcal{D}$ as follows: for each pair $R_{0}^{\prime}, R_{0}^{\prime \prime} \in \mathcal{R}(A), R_{0}^{\prime} \geq_{R_{0}} R_{0}^{\prime \prime}$ if there is a path from $R_{0}$ to $R_{0}^{\prime \prime}$ in $\mathcal{D}$ without restoration containing $R_{0}^{\prime}$. The asymmetric order $>_{\boldsymbol{R}_{\mathbf{0}}}$ associated with $\geq_{R_{0}}$ is defined in the obvious way. If $R_{0}$ is the truth and $R_{0}^{\prime}>_{R_{0}} R_{0}^{\prime \prime}$, then $R_{0}^{\prime}$ is a smaller lie than $R_{0}^{\prime \prime}$ in the following sense: in order to obtain $R_{0}^{\prime \prime}$ from $R_{0}^{\prime}$, we need to switch several pairs of objects whose rankings are adjacent; each switching is a lie according to $R_{0}$ and is added to existing lies. It is easy to check that $\geq_{R_{0}}$ is reflexive, anti-symmetric, and transitive, so that $\left(\mathcal{D}, \geq_{R_{0}}\right)$ is a partially ordered set. The following property requires that for each $i \in N$, when lies are measured by $\geq_{R_{i}}$, agent $i$ 's welfare, as measured by $R_{i}^{e}$, weakly decrease as he reports increasingly bigger lies. 
e-Lie monotonicity: For each $R \in \mathcal{D}^{N}$ and each $i \in N$, the function $\varphi_{i}\left(\cdot, R_{-i}\right):\left(\mathcal{D}, \geq_{R_{i}}\right) \rightarrow$ $\left(\Delta A, R_{i}^{e}\right)$ is monotonic; i.e., for each pair $R_{i}^{\prime}, R_{i}^{\prime \prime} \in \mathcal{D}$ such that $R_{i}^{\prime} \geq_{R_{i}} R_{i}^{\prime \prime}, \varphi_{i}\left(R_{i}^{\prime}, R_{-i}\right) R_{i}^{e} \varphi_{i}\left(R_{i}^{\prime \prime}, R_{-i}\right)$.

Example 4. Illustrating lie monotonicity. Let $A \equiv\{1,2,3,4\}$ and refer to Figure 2, Consider the universal domain $\mathcal{R}(A)$. Let $R \in \mathcal{R}(A)^{N}$ and $i \in N$. Suppose that $R_{i} \equiv 1234$. Consider a path $\{1234,1243,1423,1432,4132,4312,4321\}$ (at the top of the figure) from 1234 to 4321 in $\mathcal{R}(A)$. Since the path is without restoration, it is completely ordered by $\geq_{R_{i}}$. Let $e \in\{s d, d l, u l\}$. Suppose that agent $i$ reports preference relations in the path, sequentially, starting from 1234 . Then $e$-lie monotonicity requires that agent $i$ 's welfare, as measured by $R_{i}^{e}$, weakly decrease. In the case of the $s d$-extension, this, in particular, implies comparability of all welfare levels attained along the path.

In general, e-lie monotonicity is stronger than e-strategy-proofness. But for each $e \in\{s d, d l, u l\}$, under various domain assumptions, the two are equivalent.

Theorem 2. Let $\mathcal{D}$ be a connected domain.

(1) If $\mathcal{D}$ satisfies the non-restoration property, then $s d$-strategy-proofness is equivalent to sd-lie monotonicity.

(2) If $\mathcal{D}$ satisfies the POF path property, then dl-strategy-proofness is equivalent to dl-lie monotonicity.

(3) If $\mathcal{D}$ satisfies the LOF path property, then ul-strategy-proofness is equivalent to ul-lie monotonicity.

Proof. We only prove part (1); a similar argument applies to parts (2) and (3). Let $\varphi$ be $s d$ strategy-proof. Let $R \in \mathcal{D}^{N}$ and $i \in N$. Let $R_{i}^{\prime}, R_{i}^{\prime \prime} \in \mathcal{D}$ be such that $R_{i}^{\prime} \geq_{R_{i}} R_{i}^{\prime \prime}$. We may assume that $R_{i}^{\prime} \neq R_{i}^{\prime \prime}$, so that $R_{i}^{\prime}>_{R_{i}} R_{i}^{\prime \prime}$. Then there is be a path $\left\{R_{i}^{0}, R_{i}^{1}, \cdots, R_{i}^{m}, \cdots, R_{i}^{h}\right\}$ from $R_{i}$ to $R_{i}^{\prime \prime}$ in $\mathcal{D}$ without restoration containing $R_{i}^{\prime}$ such that $R_{i}^{m}=R_{i}^{\prime}$. For each $\tilde{h} \in\{0,1, \cdots, h\}$, let $\pi_{i}^{\tilde{h}} \equiv \varphi_{i}\left(R_{i}^{\tilde{h}}, R_{-i}\right)$.

Consider $\pi_{i}^{m}$ and $\pi_{i}^{m+1}$. Since $R_{i}^{m}$ and $R_{i}^{m+1}$ are adjacent, there are exactly two objects $k, k^{\prime} \in A$ such that $k P_{i}^{m} k^{\prime}$ and $k^{\prime} P_{i}^{m+1} k$. Since the path $\left\{R_{i}^{0}, R_{i}^{1}, \cdots, R_{i}^{m}, \cdots, R_{i}^{h}\right\}$ is without restoration, it follows that $k P_{i} k^{\prime}$ and $k^{\prime} P_{i}^{\prime \prime} k$. Since $\mathcal{D}$ is connected and satisfies the non-restoration property, the characterization of $s d$-strategy-proofness in Remark 2 implies that either (i) $\pi_{i}^{m}=\pi_{i}^{m+1}$; or (ii) $\pi_{i k}^{m}>\pi_{i k}^{m+1}, \pi_{i k^{\prime}}^{m}<\pi_{i k^{\prime}}^{m+1}$, and for each $\ell \in A \backslash\left\{k, k^{\prime}\right\}, \pi_{i \ell}^{m}=\pi_{i \ell}^{m+1}$. Thus, $\pi_{i}^{m} R_{i}^{s d} \pi_{i}^{m+1}$.

It is clear that for each $\tilde{h} \in\{m+1, \cdots, h\}$, the previous argument can be adapted to $\pi_{i}^{\tilde{h}}$ and $\pi_{i}^{\tilde{h}+1}$, showing that $\pi_{i}^{\tilde{h}} R_{i}^{s d} \pi_{i}^{\tilde{h}+1}$. Thus, $\pi_{i}^{m} R_{i}^{s d} \pi_{i}^{h}$.

Remark 3. Haeringer and Halaburda (2014) show that (i) for deterministic rules, e-strategyproofness is equivalent to e-lie monotonicity on any domain; and (ii) when probabilistic rules 
are allowed, a weakening of the non-restoration property, ensures the equivalence of sd-strategyproofness and sd-lie monotonicity. However, even the non-restoration property does not generalize to the other extensions (see Example 5 below). The advantage of our approach is that the equivalence of sd-strategy-proofness and sd-lie monotonicity follows as a corollary to the characterization of e-strategy-proofness in Remark 2 .

Example 5. Insufficiency of the non-restoration property for the $\mathrm{dl}$ - and ul-extensions. Let $N \equiv\{1,2,3\}, A \equiv\{1,2,3\}$, and $\mathcal{D} \equiv\{123,213,231,321\}$. Note that $\mathcal{D}$ is connected, and satisfies the non-restoration property but not the POF path property. Define a rule $\varphi$ as follows. For each $R_{-1} \in \mathcal{D}^{N \backslash\{1\}}$, let $\varphi_{1}\left(123, R_{-1}\right)=(0.3,0.4,0.3) ; \varphi_{1}\left(213, R_{-1}\right)=(0.2,0.5,0.3)$; $\varphi_{1}\left(231, R_{-1}\right)=(0.2,0.5,0.3) ; \varphi_{1}\left(321, R_{-1}\right)=(0.3,0.3,0.4)$. Also, for each $R \in \mathcal{D}^{N}, \varphi_{2}(R)=$ $\varphi_{3}(R)=\frac{1}{2}\left[(1,1,1)-\varphi_{1}(R)\right]$. Then $\varphi$ is dl-strategy-proof. Let $R_{1} \equiv 123$. Then $231>_{R_{1}} 321$, but for any $R_{-1} \in \mathcal{D}^{N \backslash\{1\}}, \varphi_{1}\left(321, R_{-1}\right) P_{1}^{\text {dl }} \varphi_{1}\left(231, R_{-1}\right)$. Thus, $\varphi$ is not dl-lie monotonic.

Next, let $\hat{\mathcal{D}} \equiv\{123,132,312,321\}$. Then $\hat{\mathcal{D}}$ is connected, and satisfies the non-restoration property but not the LOF path property. Define a rule $\hat{\varphi}$ that is similar to $\varphi$ except for the following. For each $R_{-1} \in \mathcal{D}^{N \backslash\{1\}}$, let $\hat{\varphi}_{1}\left(123, R_{-1}\right)=(0.3,0.4,0.3) ; \hat{\varphi}_{1}\left(132, R_{-1}\right)=(0.3,0.3,0.4)$; $\hat{\varphi}_{1}\left(312, R_{-1}\right)=(0.3,0.3,0.4) ; \hat{\varphi}_{1}\left(321, R_{-1}\right)=(0.2,0.5,0.3)$. Then $\hat{\varphi}$ is ul-strategy-proof. Let $R_{1} \equiv 123$. Then $312>_{R_{1}} 321$, but for any $R_{-1} \in \mathcal{D}^{N \backslash\{1\}}, \varphi_{1}\left(321, R_{-1}\right) P_{1}^{d l} \varphi_{1}\left(312, R_{-1}\right)$. Thus, $\varphi$ is not ul-lie monotonic.

Theorem 1 allows us to "decompose" sd-strategy-proofness into two substantially weaker strategic requirements. When there are just three objects, it follows by definition that sd-strategyproofness is equivalent to the combination of dl-strategy-proofness and ul-strategy-proofness. On the other hand, with three or more objects, we cannot deduce the equivalence directly from the definition. However, the characterization of e-strategy-proofness in Remark 2 reveals that the equivalence still holds. We omit a simple proof of this result.

Theorem 3. Let $\mathcal{D}$ be a connected domain satisfying the POF and LOF path properties. Then $s d$-strategy-proofness is equivalent to the combination of $d l$-strategy-proofness and ul-strategyproofness.

To see why this result is unexpected, let $\alpha \in(0,1)$. Consider an agent with vNM preferences $\left(1, \alpha, \alpha^{2}, \cdots, \alpha^{n-1}\right)$, which attach utility 1 to his most preferred object, utility $\alpha$ to his second most preferred object, and so on. As $\alpha \rightarrow 0^{+}$, his preferences get closer to the preferences obtained by the $d l$-extension, and $d l$-strategy-proofness requires that no agent with such extreme vNM preferences gain by lying. A symmetric argument applies to ul-strategy-proofness. Consider an agent with vNM preferences $\left(-\alpha^{n-1}, \cdots,-\alpha^{2},-\alpha,-1\right)$. As $\alpha \rightarrow 0^{+}$, his preferences get closer 
to the preferences obtained by the ul-extension, and ul-strategy-proofness requires that no agent with such extreme vNM preferences gain by lying. Thus, when $d l$ - and ul-strategy-proofness are imposed, we require immunity to manipulation by agents with those two types of extreme preferences. This is significantly weaker than sd-strategy-proofness. The latter requires that no agent with any preferences over lotteries - vNM type or not-gain by lying.

Theorem 3 relies on the characterization of e-strategy-proofness, which in turn relies on the equivalence of e-adjacent strategy-proofness and e-strategy-proofness. On the domains where the latter equivalence no longer holds, Theorem 3 also fails. The following example illustrates this point.

Example 6. The combination of $d l$-strategy-proofness and ul-strategy-proofness may not imply $s d$-strategy-proofness. Let $N \equiv\{1,2,3,4\}, A \equiv\{1,2,3,4\}$, and $\mathcal{D} \equiv\{1234,4321\}$. Note that $\mathcal{D}$ does not satisfy connectedness, which is necessary for the equivalence of e-adjacent strategyproofness and e-strategy-proofness for each $e \in\{s d, d l, u l\}$. Define a rule $\varphi$ as follows. For each $R_{-1} \in \mathcal{D}^{N \backslash\{1\}}$, let $\varphi_{1}\left(1234, R_{-1}\right)=(0.2,0.2,0.6,0)$ and $\varphi_{1}\left(4321, R_{-1}\right)=(0,0.6,0.2,0.2)$. Also, for each $R \in \mathcal{D}^{N}$ and each $i \in N \backslash\{1\}, \varphi_{i}(R)=\frac{1}{4}\left[(1,1,1,1)-\varphi_{1}(R)\right]$. Clearly, $\varphi$ is $d l$ - and ul-strategyproof. But for any $R_{-1} \in \mathcal{D}^{N \backslash\{1\}}$, it is not the case that $\varphi_{1}\left(1234, R_{-1}\right)(1234)^{s d} \varphi_{1}\left(4321, R_{-1}\right)$. Thus, $\varphi$ is not sd-strategy-proof.

Finally, we consider another weakening of strategy-proofness studied in the literature. When checking e-strategy-proofness, if agent $i$ with true preference relation $R_{i}$ reports $R_{i}^{\prime}$, we require that $\varphi_{i}(R) R_{i}^{e} \varphi_{i}\left(R_{i}^{\prime}, R_{-i}\right)$. If $R_{i}^{e}$ is not a complete relation, the latter requirement may be violated for two reasons: (i) $\varphi_{i}\left(R_{i}^{\prime}, R_{-i}\right) P_{i}^{e} \varphi_{i}(R)$; or (ii) $\varphi_{i}(R)$ and $\varphi_{i}\left(R_{i}^{\prime}, R_{-i}\right)$ are not comparable according to $R_{i}^{e}$. A number of authors study the following property that only excludes (i) (e.g., Bogomolnaia and Moulin, 2001; Kojima, 2009; Aziz, Brandl, and Brandt, 2014).

$\boldsymbol{e}$-Weak strategy-proofness: For each $R \in \mathcal{D}^{N}$, each $i \in N$, and each $R_{i}^{\prime} \in \mathcal{D}$, if $\varphi_{i}\left(R_{i}^{\prime}, R_{-i}\right) R_{i}^{e} \varphi_{i}(R)$, then $\varphi_{i}(R) I_{i}^{e} \varphi_{i}\left(R_{i}^{\prime}, R_{-i}\right)$.

Let $e$ and $\hat{e}$ be extensions such that $e \subseteq \hat{e}$. Then by definition, $\hat{e}$-weak strategy-proofness implies $e$-weak strategy-proofness. Since $s d \subsetneq d l$ and $s d \subsetneq u l$, we obtain the following observation.

Proposition 1. For each $e \in\{d l, u l\}$, the following logical relations hold: sd-strategy-proofness $\Longrightarrow e$-strategy-proofness $=e$-weak strategy-proofness $\Longrightarrow s d$-weak -strategy-proofness.

\section{$5 \quad$ Efficiency and No-envy}

Our next axiom is efficiency. Given an economy $R \in \mathcal{R}(A)^{N}$ and assignments $\pi, \pi^{\prime} \in \Pi, \boldsymbol{\pi} \boldsymbol{e}$ Pareto dominates $\boldsymbol{\pi}^{\prime}$ for $\boldsymbol{R}$ if (i) for each $i \in N, \pi_{i} R_{i}^{e} \pi_{i}^{\prime}$; and (ii) for some $i \in N, \pi_{i} P_{i}^{e} \pi_{i}^{\prime}$. An 
assignment is $\boldsymbol{e}$-efficient for $\boldsymbol{R}$ if no other assignment $e$-Pareto dominates it for $R$. The following axiom requires that for each economy, a rule select an e-efficient assignment.

e-Efficiency: For each $R \in \mathcal{R}(A)^{N}, \varphi(R)$ is e-efficient for $R$.

Now we examine how efficiency notions based on different extensions are related. Let $e$ and $\hat{e}$ be extensions such that $e \subseteq \hat{e}$. By definition, $\hat{e}$-efficiency implies e-efficiency. Applying this observation to the fact that $s d \subsetneq d l$ and $s d \subsetneq u l$, it follows that sd-efficiency is weaker than $d l$-efficiency and ul-efficiency. However, our result below shows that in fact, the three notions are equivalent. We prove the equivalence by showing that the three notions are characterized by the same condition on a binary relation over $A$.

For each $R \in \mathcal{R}(A)^{N}$ and each $\pi \in \Pi$, define a binary relation $\boldsymbol{\tau}(\boldsymbol{R}, \boldsymbol{\pi})$ over $A$ as follows: for each pair $k, \ell \in A, k \tau(R, \pi) \ell$ if there is $i \in N$ such that $k P_{i} \ell$ and $\pi_{i \ell}>0$. The relation $\tau(R, \pi)$ is cyclic if there are $k_{1}, \cdots, k_{m} \in A$ such that $k_{1} \tau(R, \pi) k_{2} \tau(R, \pi) \cdots \tau(R, \pi) k_{m} \tau(R, \pi) k_{1}$; and $\tau(R, \pi)$ is acyclic if it is not cyclic. Bogomolnaia and Moulin (2001) show that sd-efficiency is equivalent to the acyclicity of $\tau(\cdot, \cdot)$. However, this characterization is not limited to sd-efficiency.

Theorem 4. Let $e \in\{s d, d l, u l\}$. For each $R \in \mathcal{R}(A)^{N}$ and each $\pi \in \Pi, \pi$ is e-efficient for $R$ if and only if $\tau(R, \pi)$ is acyclic. Thus, sd-efficiency, dl-efficiency, and ul-efficiency are equivalent.

Proof. We omit the simple proof of the "only if" part. To prove the "if" part, let $e \in\{s d, d l, u l\}$. Let $R \in \mathcal{R}(A)^{N}$ and $\pi \in \Pi$. Assume that $\tau \equiv \tau(R, \pi)$ is acyclic. Suppose, by contradiction, that there is $\pi^{\prime} \in \Pi$ such that for each $i \in N, \pi_{i}^{\prime} R_{i}^{e} \pi_{i}$, and for some $i_{1} \in N, \pi_{i_{1}}^{\prime} P_{i_{1}}^{e} \pi_{i_{1}}$. We distinguish two cases (if $e=s d$, then the argument in either case suffices).

Case 1: $e=d l$.

By the definition of the $d l$-extension, there are $k_{1}, k_{2} \in A$ such that $k_{2} P_{i_{1}} k_{1}, \pi_{i_{1} k_{1}}>\pi_{i_{1} k_{1}}^{\prime}$, and $\pi_{i_{1} k_{2}}<\pi_{i_{1} k_{2}}^{\prime}$. Thus, $\pi_{i_{1} k_{1}}>0$, so that $k_{2} \tau k_{1}$. Now because $\pi_{i_{1} k_{2}}<\pi_{i_{1} k_{2}}^{\prime}$, by feasibility, there is $i_{2} \in N$ such that $\pi_{i_{2} k_{2}}>\pi_{i_{2} k_{2}}^{\prime}$. This implies, in particular, that $\pi_{i_{2}} \neq \pi_{i_{2}}^{\prime}$. Since $R_{i_{2}}^{d l}$ is anti-symmetric, $\pi_{i_{2}}^{\prime} P_{i_{2}}^{d l} \pi_{i_{2}}$. Again, by the definition of the $d l$-extension, there is $k_{3} \in A$ such that $k_{3} P_{i_{2}} k_{2}$ and $\pi_{i_{2} k_{3}}<\pi_{i_{2} k_{3}}^{\prime}$. Thus, because $\pi_{i_{2} k_{2}}>0, k_{3} \tau k_{2}$. Continuing this process, by finiteness of $A$, we can construct a cycle of $\tau$, a contradiction.

Case 2: $e=u l$.

By the definition of the $u l$-extension, there are $k_{1}, k_{2} \in A$ such that $k_{1} P_{i_{1}} k_{2}, \pi_{i_{1} k_{1}}<\pi_{i_{1} k_{1}}^{\prime}$, and $\pi_{i_{1} k_{2}}>\pi_{i_{1} k_{2}}^{\prime}$. Thus, $\pi_{i_{1} k_{2}}>0$, so that $k_{1} \tau k_{2}$. Now because $\pi_{i_{1} k_{2}}>\pi_{i_{1} k_{2}}^{\prime}$, by feasibility, there is $i_{2} \in N$ such that $\pi_{i_{2} k_{2}}<\pi_{i_{2} k_{2}}^{\prime}$. This implies, in particular, that $\pi_{i_{2}} \neq \pi_{i_{2}}^{\prime}$. Since $R_{i_{2}}^{u l}$ is anti-symmetric, $\pi_{i_{2}}^{\prime} P_{i_{2}}^{u l} \pi_{i_{2}}$. Again, by the definition of the $u l$-extension, there is $k_{3} \in A$ such that $k_{2} P_{i_{2}} k_{3}$ and $\pi_{i_{2} k_{3}}>\pi_{i_{2} k_{3}}^{\prime}$. Thus, $\pi_{i_{2} k_{3}}>0$, so that $k_{2} \tau k_{3}$. Continuing this process, by finiteness of $A$, we can construct a cycle of $\tau$, a contradiction. 
Several papers study sd-efficiency. McLennan (2002) establishes the "ordinal welfare theorem": an assignment $\pi$ is sd-efficient for an economy $R$ if and only if there is a profile of vNM utility functions $u \equiv\left(u_{i}\right)_{i \in N}$ such that (i) for each $i \in N, u_{i}$ is consistent with $R_{i}$; and (ii) $\pi$ is Pareto efficient for $u$. Abdulkadiroğlu and Sönmez (2003a) characterize sd-efficiency by a dominance notion defined over sets of deterministic assignments. Liu and Pycia (2012) prove a result that relate efficiency for deterministic assignments with sd-efficiency in the limit: each sequence of rules consisting of uniform randomizations over efficient deterministic rules is asymptotically sd-efficient. Since $s d-, d l$-, and $u l$-efficiency are equivalent, these results also apply to $d l$-and $u l$-efficiency.

Next is a fairness axiom that originates in Tinbergen (1953) and Foley (1967). It says that no agent should prefer someone else's lottery to his own.

e-No-envy: For each $R \in \mathcal{R}(A)^{N}$ and each pair $i, j \in N, \varphi_{i}(R) R_{i}^{e} \varphi_{j}(R)$.

As is the case for e-strategy-proofness, when $R_{i}^{e}$ is not complete, e-no-envy may be violated because lotteries are not comparable. The following axiom relaxes e-no-envy by allowing for such cases: no agent prefers someone else's lottery to his own.

e-Weak no-envy: For each $R \in \mathcal{R}(A)^{N}$ and each pair $i, j \in N$, if $\varphi_{j}(R) R_{i}^{e} \varphi_{i}(R)$, then $\varphi_{i}(R) I_{i}^{e} \varphi_{j}(R)$.

Next, we study logical relations among various notions of no-envy. Let $e$ and $\hat{e}$ be extensions such that $e \subseteq \hat{e}$. Then by definition, e-no-envy implies $\hat{e}-n o-e n v y$ and $\hat{e}$-weak no-envy implies e-weak no-envy. Thus, we obtain the following.

Proposition 2. For each $e \in\{d l, u l\}$, the following logical relations hold:

$s d$-no-envy $\Longrightarrow e$-no-envy $=e$-weak no-envy $\Longrightarrow e$-weak no-envy.

\section{The Generalized Serial and Random Priority Rules}

In this section, we define the serial and random priority rules, introduce a family of rules that generalize the serial rule, and assess them in terms of efficiency, no-envy, and strategy-proofness. The serial rule (Bogomolnaia and Moulin, 2001), denoted $\boldsymbol{S}$, is defined by a simultaneous consumption algorithm, which works as follows. Each object is treated as a continuum of measure 1, consisting of "(probability) shares" of the object. Shares of the objects are distributed over an imaginary time horizon. At time $t=0$, each agent starts consuming shares of his most preferred object at unit speed. When an object is "exhausted", i.e., all of its shares are distributed, each agent who has consumed the object and whose total consumption is less than 1 moves on to his next most preferred object and consumes its shares until it is exhausted, and so on. The algorithm terminates when each agent's consumption reaches 1. Collecting the information on each agent's consumption of all objects, we obtain an assignment. The serial rule selects this assignment. 
Next is the random priority rule. First, fix a priority order over the agents $b: N \rightarrow\{1, \cdots, n\}$ (for each pair $i, j \in N$, if $b(i)<b(j)$, then agent $i$ has a higher priority than agent $j$ ). The sequential priority rule associated with priority order $\boldsymbol{b}$, denoted $\boldsymbol{S P}^{\boldsymbol{b}}$, assigns objects as follows: first, the agent with the highest priority chooses his most preferred object; then the agent with the second highest priority chooses his most preferred object among those available to him, and so on. Since $n$ ! priority orders are possible, there are $n$ ! sequential priority rules. The random priority rule, denoted $\boldsymbol{R P}$, is an average of the sequential priority rules. That is, letting $B$ be the set of all priority orders, for each $R \in \mathcal{R}(A)^{N}, R P(R) \equiv \frac{1}{n !} \sum_{b \in B} S P^{b}(R)$.

Two properties of the serial rule are notable: in allocating objects, the names of agents do not matter - anonymity - and neither do the names of objects - neutrality. But a mechanism designer may wish to discard neutrality and favor particular preferences, without violating anonymity. Consider, for instance, the problem of allocating on-campus housing units. Let us assume that each housing unit is either a single or double room. If single rooms are more popular, the designer may want to promote double rooms by assigning them, with a high probability, to students who prefer them. Such asymmetric treatment of students is based on preferences, not identity. The serial rule cannot serve this purpose because it is neutral. Thus, we generalize it by allowing the speed at which probability shares are distributed to vary across objects.

An (allocation) speed function is a mapping $\sigma: A \times \mathbb{R}_{+} \rightarrow \mathbb{R}_{++}$such that for each $k \in A$, (i) $\sigma(k, \cdot)$ is measurable; and (ii) for each $t \in \mathbb{R}_{+}$, there is $\bar{t}<\infty$ satisfying $\int_{t}^{\bar{t}} \sigma(k, \tau) d \tau=1$. Let $\Sigma$ be the set of all such functions. Let $\sigma \in \Sigma$. We define the generalized serial rule associated with $\boldsymbol{\sigma}$, denoted $\boldsymbol{S}^{\boldsymbol{\sigma}}$, by the generalized simultaneous consumption algorithm associated with $\boldsymbol{\sigma}$. To illustrate it, we again treat each object as a continuum of measure 1 , consisting of shares of the object. At time $t=0$, each agent goes to his most preferred object. For each $(k, t) \in A \times \mathbb{R}_{+}$, at time $t$, shares of object $k$ are allocated at speed $\sigma(k, t)$ to those agents who consume object $k$. Each agent consumes his most preferred object until either his total consumption of shares reaches 1 or his most preferred object is exhausted. In the former case, the agent is removed; and in the latter, the object is removed (the two cases may happen at the same time). Then we continue the consumption process with the remaining agents and objects. Each remaining agent moves on to the object he most prefers among those remaining. The process terminates when each agent's consumption reaches 1 . Note that for each $(k, t) \in A \times \mathbb{R}_{+}$, the speed $\sigma(k, t)$ of object $k$ at time $t$ is independent of the identity of the agents.

To define the algorithm formally, for each $R \in \mathcal{R}(A)^{N}$, each non-empty $M \subseteq N$, each nonempty $B \subseteq A$, and each $k \in B$, let $\boldsymbol{N}^{*}(\boldsymbol{R}, \boldsymbol{M}, \boldsymbol{B}, \boldsymbol{k}) \equiv\left\{i \in M\right.$ : for each $\left.\ell \in B, k R_{i} \ell\right\}$. Let $N^{0} \equiv N, A^{0} \equiv A, t^{0} \equiv 0$, and $\pi^{0} \equiv(0)_{i \in N, k \in A}$. For each $s \in \mathbb{N}$, given $\left(N^{s-1}, A^{s-1}, t^{s-1}, \pi^{s-1}\right)$, define $\left(N^{s}, A^{s}, t^{s}, \pi^{s}\right)$ recursively as follows. For each $i \in N^{s-1}$, denoting by $k$ the object agent $i$ 
most prefers in $A^{s-1}$, let

$$
t^{s}(i) \equiv \inf \left\{t \in \mathbb{R}_{+}: \int_{t^{s-1}}^{t} \sigma(k, \tau) d \tau+\sum_{\ell \in A} \pi_{i \ell}^{s-1}=1\right\}
$$

For each $k \in A^{s-1}$, let

$$
t^{s}(k) \equiv \inf \left\{t \in \mathbb{R}_{+}:\left|N^{*}\left(R, N^{s-1}, A^{s-1}, k\right)\right| \cdot \int_{t^{s-1}}^{t} \sigma(k, \tau) d \tau+\sum_{i \in N} \pi_{i k}^{s-1}=1\right\}
$$

if $N^{*}\left(R, N^{s-1}, A^{s-1}, k\right) \neq \emptyset$; and $t^{s}(k) \equiv \infty$ otherwise. Let $t^{s} \equiv \min _{h \in N^{s-1} \cup A^{s-1}} t^{s}(h) ; N^{s} \equiv$ $N^{s-1} \backslash\left\{i \in N^{s-1}: t^{s}(i)=t^{s}\right\} ; A^{s} \equiv A^{s-1} \backslash\left\{k \in A^{s-1}: t^{s}(k)=t^{s}\right\} ;$ and $\pi^{s} \equiv\left(\pi_{i k}^{s}\right)_{i \in N, k \in A}$, where for each $i \in N$ and each $k \in A$,

$$
\pi_{i k}^{s} \equiv \begin{cases}\pi_{i k}^{s-1}+\int_{t^{s-1}}^{t^{s}} \sigma(k, t) d t & \text { if } i \in N^{*}\left(R, N^{s-1}, A^{s-1}, k\right) \\ \pi_{i k}^{s-1} & \text { otherwise. }\end{cases}
$$

By condition (ii) in the definition of speed functions, it follows that for each $s \in \mathbb{N}, t^{s}<\infty$. Also, for each $s \in \mathbb{N}, N^{s-1} \supseteq N^{s}$ and $A^{s-1} \supseteq A^{s}$, with at least one of the inclusions holding strictly. Thus, there is $\hat{s} \in \mathbb{N}$ such that $N^{\hat{s}}=\emptyset$ and $A^{\hat{s}}=\emptyset$. Then $\pi^{\hat{s}} \in \Pi$, and $S^{\sigma}$ chooses $\pi^{\hat{s}}$ for $R$; i.e., $S^{\sigma}(R)=\pi^{\hat{s}}$.

Now we assess the family of generalized serial rules and the random priority rule based on the axioms in Sections 445. The first criterion is efficiency. While the random priority rule is not sd-efficient, for each $\sigma \in \Sigma$, the generalized serial rule associated with $\sigma$ is sd-efficient. Further, since $s d-, d l-$, and $u l$-efficiency are equivalent, we can state the (in)efficiency of these rules in more general terms.

Theorem 5. (1) For each $e \in\{s d, d l, u l\}$ and each $\sigma \in \Sigma$, the generalized serial rule associated with $\sigma$ is e-efficient.

(2) For each $e \in\{s d, d l, u l\}$, the random priority rule is not e-efficient.

Proof. We only prove part (1); part (2) follows from Theorem 4 and the fact that the random priority rule is not $s d$-efficient (Bogomolnaia and Moulin, 2001). Let $e \in\{s d, d l, u l\}$ and $\sigma \in$ $\Sigma$. Let $R \in \mathcal{R}(A)^{N}$ and $\pi \equiv S^{\sigma}(R)$. By Theorem 4 it suffices to show that $\tau \equiv \tau(R, \pi)$ is acyclic. Suppose, by contradiction, that $\tau$ is cyclic; i.e., there are $k_{1}, \cdots, k_{m} \in A$ such that $k_{1} \tau k_{2} \tau \cdots \tau k_{m} \tau k_{1}$. For each $h \in\{1, \cdots, m\}$, there is $i_{h} \in N$ such that $k_{h} P_{i_{h}} k_{h+1}$ and $\pi_{i_{h} k_{h+1}}>$ 0 (with the convention that $k_{m+1}=k_{1}$ ). Now consider the generalized simultaneous consumption algorithm associated with $\sigma$, applied to $R$. For each $h \in\{1, \cdots, m\}$, let $s_{h+1}$ be the first step $s$ 
in the algorithm in which agent $i_{h}$ consumes object $k_{h+1}$ (i.e., smallest $s$ such that $\pi_{i_{h} k_{h+1}}^{s}>0$ ). Let $h \in\{1, \cdots, m\}$. Note that step $s_{h+1}$ begins with the objects in $A^{s_{h+1}-1}$ and the agents in $N^{s_{h+1}-1}$. Since $k_{h} P_{i_{h}} k_{h+1}$ and agent $i_{h}$ does not consume object $k_{h}$ in step $s_{h+1}$, it follows that $k_{h} \notin A^{s_{h+1}-1}$. Thus, $s_{h}<s_{h+1}$. Then $s_{1}<s_{2}<\cdots<s_{m}<s_{1}$, a contradiction.

Remark 4. While our model is a fixed population framework, we can state the inefficiency of the random priority rule in stronger terms by allowing $n$, the common number of agents and objects, to approach infinity. Combined with part (2) of Theorem 5, Manea (2009, Theorem 1) yields the following: for each $e \in\{s d, d l, u l\}$, the fraction of economies for which the random priority rule selects an $e$-efficient assignment converges to zero as $n \rightarrow \infty$.

Next is no-envy. The serial rule satisfies sd-no-envy (Bogomolnaia and Moulin, 2001). Since for each $e \in\{d l, u l\}$, sd-no-envy implies e-no-envy, the rule satisfies the strongest no-envy concept. On the other hand, the generalized serial rules violate $s d$-no-envy in general (see Example 7 below), and the same is true for the random priority rule. The strongest they satisfy is dl-no-envy.

Theorem 6. (1) For each $\sigma \in \Sigma$, the generalized serial rule associated with $\sigma$ satisfies dl-no-envy.

(2) The random priority rule satisfies dl-no-envy.

Proof. Part (1). Let $\sigma \in \Sigma, R \in \mathcal{R}(A)^{N}$, and $i, j \in N$ with $i \neq j$. Assume, without loss of generality, that $1 P_{i} 2 P_{i} \cdots P_{i} n$. Let $\pi \equiv S^{\sigma}(R)$. To show that $\pi_{i} R_{i}^{d l} \pi_{j}$, consider the generalized simultaneous consumption algorithm associated with $\sigma$, applied to $R$. Let $s_{1}$ be the step in which object 1 is exhausted; i.e., $s_{1}$ is such that $1 \in A^{s_{1}-1} \backslash A^{s_{1}}$

Now we show that $\pi_{i 1} \geq \pi_{j 1}$. First, for each $s \leq s_{1}-1, i \in N^{*}\left(R, N^{s}, A^{s}, 1\right)$. Also, there is $\hat{t} \in\left[0, t^{s_{1}}\right]$ such that agent $j$ consumes object 1 during the interval $\left[\hat{t}, t^{s_{1}}\right)$. Thus,

$$
\pi_{i 1}^{s_{1}}=\int_{0}^{t^{s_{1}}} \sigma(1, t) d t \geq \int_{\hat{t}}^{t^{s_{1}}} \sigma(1, t) d t=\pi_{j 1}^{s_{1}}
$$

Moreover, because object 1 is exhausted in Step $s_{1}, \pi_{i 1}=\pi_{i 1}^{s_{1}}$ and $\pi_{j 1}=\pi_{j 1}^{s_{1}}$, so that $\pi_{i 1} \geq \pi_{j 1}$.

If $\pi_{i 1}>\pi_{j 1}$, then $\pi_{i} P_{i}^{d l} \pi_{j}$. Assume, henceforth, that $\pi_{i 1}=\pi_{j 1}$. Since for each $t \in \mathbb{R}_{+}$, $\sigma(1, t)>0$, Inequality (1) implies that in fact, $\hat{t}=0$, so that for each $k \in A, 1 R_{j} k$. Now let $s_{2}$ be the step in which the objects in $\{1,2\}$ are exhausted; i.e., $s_{2}$ is such that $A^{s_{2}-1} \cap\{1,2\} \neq \emptyset$ and $A^{s_{2}} \cap\{1,2\}=\emptyset$.

To show that $\pi_{i 2} \geq \pi_{j 2}$, note that $s_{1} \leq s_{2}$. If $s_{1}=s_{2}$, then $\pi_{i 2}=\pi_{j 2}=0$. If $s_{1}<s_{2}$, then for each $s \in\left\{s_{1}, s_{1}+1, \cdots, s_{2}-1\right\}, i \in N^{*}\left(R, N^{s}, A^{s}, 2\right)$. Also, there is $t^{\prime} \in\left[t^{s_{1}}, t^{s_{2}}\right]$ such that agent

\footnotetext{
${ }^{18}$ Note that Step $s_{1}$ begins with the objects in $A^{s_{1}-1}$ and the agents in $N^{s_{1}-1}$.
} 
$j$ consumes object 2 during the interval $\left[t^{\prime}, t^{s_{2}}\right)$. Thus,

$$
\pi_{i 2}^{s_{2}}=\int_{t^{s_{1}}}^{t^{s_{2}}} \sigma(2, t) d t \geq \int_{t^{\prime}}^{t^{s_{2}}} \sigma(2, t) d t=\pi_{j 2}^{s_{2}}
$$

Moreover, because object 2 is exhausted in Step $s_{2}, \pi_{i 2}=\pi_{i 2}^{s_{2}}$ and $\pi_{j 2}=\pi_{j 2}^{s_{2}}$, so that $\pi_{i 2} \geq \pi_{j 2}$.

If $\pi_{i 2}>\pi_{j 2}$, then $\pi_{i} P_{i}^{d l} \pi_{j}$. Otherwise, we can repeat the above argument to eventually obtain that $\pi_{i} R_{i}^{d l} \pi_{j}$.

Part (2). Let $R \in \mathcal{R}(A)^{N}$ and $i, j \in N$ with $i \neq j$. Assume, without loss of generality, that $1 P_{i} 2 P_{i} \cdots P_{i} n$. Let $\pi \equiv R P(R)$. Let $m \equiv \frac{n !}{2}$, and enumerate the set of all priority orders over $N$ as follows: $B=\left\{b_{1}, b_{1}^{\prime}, b_{2}, b_{2}^{\prime}, \cdots, b_{m}, b_{m}^{\prime}\right\}$, where for each $h \in\{1, \cdots, m\}$, (i) $b_{h}$ and $b_{h}^{\prime}$ differ only on the priorities of agents $i$ and $j$; and (ii) $b_{h}(i)<b_{h}(j)$ and $b_{h}^{\prime}(i)>b_{h}^{\prime}(j)$. Note that $\pi_{i}=\frac{1}{2 m} \sum_{h \in\{1, \cdots, m\}} S P_{i}^{b_{h}}(R)+S P_{i}^{b_{h}^{\prime}}(R)$ and $\pi_{j}=\frac{1}{2 m} \sum_{h \in\{1, \cdots, m\}} S P_{j}^{b_{h}}(R)+S P_{j}^{b_{h}^{\prime}}(R)$.

First, we show that $\pi_{i 1} \geq \pi_{j 1}$. It suffices to show that for each $h \in\{1, \cdots, m\}$,

$$
S P_{i 1}^{b_{h}}(R)+S P_{i 1}^{b_{h}^{\prime}}(R) \geq S P_{j 1}^{b_{h}}(R)+S P_{j 1}^{b_{h}^{\prime}}(R)
$$

Let $h \in\{1, \cdots, m\}$. By the definition of the sequential priority rules, $S P_{i 1}^{b_{h}}(R) \geq S P_{j 1}^{b_{h}^{\prime}}(R)$ and $S P_{i 1}^{b_{h}^{\prime}}(R) \geq S P_{j 1}^{b_{h}}(R)$. Thus, Inequality (3) follows.

If $\pi_{i 1}>\pi_{j 1}$, then $\pi_{i} P_{i}^{d l} \pi_{j}$. Thus, assume, henceforth, that $\pi_{i 1}=\pi_{j 1}$. Before showing that $\pi_{i 2} \geq \pi_{j 2}$, we first show that for each $k \in A, 1 R_{j} k$. Since $\pi_{i 1}=\pi_{j 1}$, for each $h \in\{1, \cdots, m\}$, Inequality (3) holds with equality. This means that for each $h \in\{1, \cdots, m\}, S P_{i 1}^{b_{h}}(R)=S P_{j 1}^{b_{h}^{\prime}}(R)$ and $S P_{i 1}^{b_{h}^{\prime}}(R)=S P_{j 1}^{b_{h}}(R)$. Thus, for each $k \in A, 1 R_{j} k$.

Next, we show that $\pi_{i 2} \geq \pi_{j 2}$. Since $\pi_{i 1}=\pi_{j 1}$, it suffices to show that for each $h \in\{1, \cdots, m\}$,

$$
\sum_{k \in\{1,2\}} S P_{i k}^{b_{h}}(R)+\sum_{k \in\{1,2\}} S P_{i k}^{b_{h}^{\prime}}(R) \geq \sum_{k \in\{1,2\}} S P_{j k}^{b_{h}}(R)+\sum_{k \in\{1,2\}} S P_{j k}^{b_{h}^{\prime}}(R)
$$

Let $h \in\{1, \cdots, m\}$. Recall that that for each $k \in A, 1 R_{j} k$. Then by the definition of the sequential priority rules, $\sum_{k \in\{1,2\}} S P_{i k}^{b_{h}}(R) \geq \sum_{k \in\{1,2\}} S P_{j k}^{b_{h}^{\prime}}(R)$ and $\sum_{k \in\{1,2\}} S P_{i k}^{b_{h}^{\prime}}(R) \geq \sum_{k \in\{1,2\}} S P_{j k}^{b_{h}}(R)$. Thus, Inequality (4) follows.

If $\pi_{i 2}>\pi_{j 2}$, then $\pi_{i} P_{i}^{d l} \pi_{j}$. If $\pi_{i 2}=\pi_{j 2}$, we can repeat the above argument to show that $\pi_{i 3} \geq \pi_{j 3}$. We omit the details.

Bogomolnaia and Moulin (2001) note that the random priority rule satisfies sd-weak no-envy, but not sd-no-envy. Theorem 6 says that it satisfies a stronger property, $d l$-no-envy. This also implies that the random priority rule violates sd-no-envy because it violates ul-no-envy. 
Example 7. A generalized serial rule may violate sd-no-envy. Let $N \equiv\{1,2,3\}$ and $A \equiv\{1,2,3\}$. Let $\varepsilon \in\left(0, \frac{1}{2}\right)$ and let $\sigma \in \Sigma$ be such that for each $t \in \mathbb{R}_{+}, \sigma(1, t)=\varepsilon$ and $\sigma(2, t)=\sigma(3, t)=1$. To show that $S^{\sigma}$ violates $s d$-no-envy, consider $R \in \mathcal{R}(A)^{N}$ such that (i) $1 P_{1} 2 P_{1} 3$; and (ii) for each $i \in\{2,3\}, 2 P_{i} 1 P_{i} 3$. Let $\pi \equiv S^{\sigma}(R)$. Then $\pi_{1}=\left(\frac{1+2 \varepsilon}{3}, 0, \frac{2-2 \varepsilon}{3}\right)$ and $\pi_{2}=\pi_{3}=\left(\frac{1-\varepsilon}{3}, \frac{1}{2}, \frac{1+2 \varepsilon}{6}\right)$. Since $\pi_{11}+\pi_{12}<\pi_{21}+\pi_{22}$, it is not the case that $\pi_{1} R_{1}^{s d} \pi_{2}$.

In terms of strategy-proofness, the random priority rule outperforms all the generalized serial rules. The former is sd-strategy-proof (Bogomolnaia and Moulin, 2001), and hence $d l$ - and $u l$ strategy-proof. By contrast, the serial rule is only dl-strategy-proof and in general, the generalized serial rules are not $d l$-strategy-proof (see Example 8 below) 19

Theorem 7. (1) The serial rule is $d l$-strategy-proof, and hence dl-lie monotonic ${ }^{20}$

(2) For each $e \in\{s d, d l, u l\}$, the random priority rule is e-strategy-proof, and hence e-lie monotonic.

While the proof of part (1) in Theorem 7 is relegated to Appendix B, we convey the main intuition informally. By Theorem 1, it is enough to verify that the serial rule is dl-adjacent strategy-proof. Suppose that agent $i$ with true preference relation $R_{i}$, say, reports a preference relation $R_{i}^{\prime}$ adjacent to $R_{i}$ while all the other agents announce $R_{-i}$. Let $k, \ell \in A$ be such that $k P_{i} \ell$ and $\ell P_{i}^{\prime} k$. Now consider the simultaneous consumption algorithm, applied to $R$ and $\left(R_{i}^{\prime}, R_{-i}\right)$. When agent $i$ changes his announcement from $R_{i}$ to $R_{i}^{\prime}$, the probability that he receives object $k$ cannot go up. And if that probability is unaffected, so is the whole lottery he receives. Thus, the serial rule is $d l$-adjacent strategy-proof.

Bogomolnaia and Moulin (2001) show that the serial rule is sd-weak strategy-proof but not sd-strategy-proof. Theorem 7 shows that indeed, it satisfies a stronger property, dl-strategyproofness. Moreover, since sd-strategy-proofness is equivalent to the combination of $d l$ - and $u l$ strategy-proofness, it follows that the serial rule violates sd-strategy-proofness because it violates ul-strategy-proofness.

Example 8. A generalized serial rule may not be $d l$-strategy-proof. Let $N \equiv\{1, \cdots, 5\}$ and $A \equiv\{1, \cdots, 5\}$. Let $\sigma \in \Sigma$ be such that (i) for each $k \in\{1,3,4,5\}$ and each $t \in \mathbb{R}_{+}, \sigma(k, t)=1$; and (ii) for each $t \in \mathbb{R}_{+}, \sigma(2, t)=\frac{1}{3}$. Let $R \in \mathcal{R}(A)^{N}$ be the economy specified in Figure 3 (the unspecified part of $R_{1}$ and $R_{2}$ can be completed in an arbitrary way). Let $\pi \equiv S^{\sigma}(R)$. It is easy to compute that $\pi_{3}=\left(0, \frac{1}{3}, \frac{1}{6}, \frac{1}{2}, 0\right)$. Now consider agent 3. Let $R_{3}^{\prime} \in \mathcal{R}(A)$ be the preference relation specified in Figure 3. Let $\pi^{\prime} \equiv S^{\sigma}\left(R_{3}^{\prime}, R_{-3}\right)$. Simple calculation shows that $\pi_{3}^{\prime}=\left(0, \frac{1}{3}, 0, \frac{1}{2}, \frac{1}{6}\right)$, so that $\pi_{3}^{\prime} P_{3}^{d l} \pi_{3}$. Thus, $S^{\sigma}$ is not dl-strategy-proof.

\footnotetext{
${ }^{19}$ I thank Jay Sethuraman for providing an example where a generalized serial rule is not $d l$-strategy-proof.

${ }^{20}$ Schulman and Vazirani (2012), independent of ours, also prove that the serial rule is dl-strategy-proof.
} 


\begin{tabular}{ccccccc}
$R_{1}$ & $R_{2}$ & $R_{3}$ & $R_{4}$ & $R_{5}$ & & $R_{3}^{\prime}$ \\
\hline 1 & 1 & 2 & 2 & 2 & & 2 \\
4 & 5 & 4 & 5 & 5 & & 5 \\
. &. & 5 & 3 & 3 & & 4 \\
. &. & 3 & 4 & 4 & & 3 \\
. &. & 1 & 1 & 1 & & 1
\end{tabular}

Figure 3: A generalized serial rule may not be $\boldsymbol{d} \boldsymbol{l}$-strategy-proof (Example 8). Let $N \equiv\{1, \cdots, 5\}$ and $A \equiv\{1, \cdots, 5\}$. Let $\sigma \in \Sigma$ be such that (i) for each $k \in\{1,3,4,5\}$ and each $t \in \mathbb{R}_{+}, \sigma(k, t)=1$; and (ii) for each $t \in \mathbb{R}_{+}, \sigma(2, t)=\frac{1}{3}$. Let $R \in \mathcal{R}(A)^{N}$ and $R_{3}^{\prime} \in \mathcal{R}(A)$ be as specified above. Let $\pi \equiv S^{\sigma}(R)$ and $\pi^{\prime} \equiv S^{\sigma}\left(R_{3}^{\prime}, R_{-3}\right)$. Since $\pi_{3}^{\prime} P_{3}^{d l} \pi_{3}, S^{\sigma}$ is not dl-strategy-proof.

We have seen that the serial rule satisfies $d l$-efficiency, $d l$-no-envy, and dl-strategy-proofness. Saban and Sethuraman (2013) show, by a counter-example, that the serial rule is not the only rule with those properties.

\section{Concluding Remarks}

The growing literature on probabilistic assignment takes the ordinal approach based on the $s d$ extension. Although the use of the $s d$-extension is well justified, taking it as the only way of extending preferences over objects to preferences over lotteries limits our analysis of assignment problems. In an attempt to complement the current practice, we introduce the $d l$ - and $u l$-extensions and re-examine standard properties of assignment rules from the perspective of all three extensions.

Some of our results are interesting in their own right; e.g., the equivalence of e-strategyproofness, e-adjacent strategy-proofness, and e-lie monotonicity for each $e \in\{s d, d l, u l\}$, and the equivalence of $s d$-, $d l$-, and ul-efficiency. But they also show that existing results (Carroll, 2012; Sato, 2013a; Haeringer and Halaburda, 2014; McLennan, 2002; Abdulkadiroğlu and Sönmez, 2003a; Liu and Pycia, 2012) do not rely on specifics of the sd-extension and are, in fact, part of a more general phenomenon. We hope that our extension approach helps uncover and address issues that are overlooked when only one extension is considered.

\section{A Appendix: Sufficiency of Adjacent Strategy-proofness}

This appendix provides a proof of Theorem 1. First, we introduce an auxiliary axiom, which we call $e$-within-m strategy-proofness, where $m \in \mathbb{N}$. It requires that no agent benefit from reporting a preference relation lying within distance $m$ (according to metric $d(\cdot, \cdot)$ ) from his true preference relation. 
$\boldsymbol{e}$-Within- $\boldsymbol{m}$ strategy-proofness: For each $R \in \mathcal{D}^{N}$, each $i \in N$, and each $R_{i}^{\prime} \in \mathcal{D}$ such that $d\left(R_{i}, R_{i}^{\prime}\right) \leq m, \varphi_{i}(R) R_{i}^{e} \varphi_{i}\left(R_{i}^{\prime}, R_{-i}\right)$.

Clearly, e-adjacent strategy-proofness and e-strategy-proofness are special cases of e-within-m strategy-proofness. In three lemmas below, we show that for each $e \in\{s d, d l, u l\}$ and each $m \in \mathbb{N}$, under the respective domain assumptions, e-within- $m$ strategy-proofness implies $e$-within- $(m+1)$ strategy-proofness. Then it follows that e-adjacent strategy-proofness implies e-strategy-proofness. We first prove the implication for the $s d$-extension.

Lemma 1. Let $\mathcal{D}$ be a connected domain satisfying the non-restoration property. Then for each $m \in \mathbb{N}$, sd-within- $m$ strategy-proofness implies $s d$-within- $(m+1)$ strategy-proofness.

Proof. Let $\mathcal{D}$ be as in the lemma. Let $\varphi$ be an sd-within-m strategy-proof rule defined on $\mathcal{D}^{N}$. Let $R \in \mathcal{D}^{N}$ and $i \in N$. Let $R_{i}^{\prime} \in \mathcal{D}$ be such that $d\left(R_{i}, R_{i}^{\prime}\right) \leq m+1$. Let $\pi_{i} \equiv \varphi_{i}(R)$ and $\pi_{i}^{\prime} \equiv \varphi_{i}\left(R_{i}^{\prime}, R_{-i}\right)$. If $d\left(R_{i}, R_{i}^{\prime}\right) \leq m$, then by sd-within-m strategy-proofness, $\pi_{i} R_{i}^{s d} \pi_{i}^{\prime}$. Thus, assume, henceforth, that $d\left(R_{i}, R_{i}^{\prime}\right)=m+1$. Suppose, without loss of generality, that $R_{i}$ is such that $1 P_{i} 2 P_{i} \cdots P_{i} n$. By the assumptions on $\mathcal{D}$, there is a path $\left\{R_{i}^{0}, R_{i}^{1}, \cdots, R_{i}^{m}, R_{i}^{m+1}\right\}$ from $R_{i}$ to $R_{i}^{\prime}$ in $\mathcal{D}$ without restoration. Let $\hat{R}_{i} \equiv R_{i}^{m}$ and $\hat{\pi}_{i} \equiv \varphi_{i}\left(\hat{R}_{i}, R_{-i}\right)$. Since $\hat{R}_{i}$ and $R_{i}^{\prime}$ are adjacent, there are exactly two objects $k_{1}, k_{2} \in A$ such that $k_{1} \hat{P}_{i} k_{2}$ and $k_{2} P_{i}^{\prime} k_{1}$. Moreover, since the path $\left\{R_{i}^{0}, R_{i}^{1}, \cdots, R_{i}^{m}, R_{i}^{m+1}\right\}$ is without restoration, $k_{1} P_{i} k_{2}$.

Since $d\left(R_{i}, \hat{R}_{i}\right)=m$, by sd-within-m strategy-proofness, $\pi_{i} R_{i}^{s d} \hat{\pi}_{i}$; i.e., for each $\ell \in A, \sum_{h \in\{1, \cdots, \ell\}} \pi_{i h} \geq$ $\sum_{h \in\{1, \cdots, \ell\}} \hat{\pi}_{i h}$. Also, by sd-within-1 strategy-proofness, $\hat{\pi}_{i} \hat{R}_{i}^{s d} \pi_{i}^{\prime}$ and $\pi_{i}^{\prime}\left(R_{i}^{\prime}\right)^{s d} \hat{\pi}_{i}$. This means that for each $\ell \in A \backslash\left\{k_{1}, k_{2}\right\}, \hat{\pi}_{i \ell}=\pi_{i \ell}^{\prime}, \hat{\pi}_{i k_{1}} \geq \pi_{i k_{1}}^{\prime}$, and $\hat{\pi}_{i k_{2}} \leq \pi_{i k_{2}}^{\prime}$. To show that $\pi_{i} R_{i}^{s d} \pi_{i}^{\prime}$, we check several inequalities as follows.

First, for each $\ell \in A$ such that $\ell P_{i} k_{1}, \hat{\pi}_{i 1}=\pi_{i 1}^{\prime}, \cdots, \hat{\pi}_{i \ell}=\pi_{i \ell}^{\prime}$, so that $\sum_{h \in\{1, \cdots, \ell\}} \pi_{i h} \geq$ $\sum_{h \in\{1, \cdots, \ell\}} \hat{\pi}_{i h}=\sum_{h \in\{1, \cdots, \ell\}} \pi_{i h}^{\prime}$.

Also, since $\hat{\pi}_{i k_{1}} \geq \pi_{i k_{1}}^{\prime}, \sum_{h \in\left\{1, \cdots, k_{1}\right\}} \pi_{i h} \geq \sum_{h \in\left\{1, \cdots, k_{1}\right\}} \hat{\pi}_{i h} \geq \sum_{h \in\left\{1, \cdots, k_{1}\right\}} \pi_{i h}^{\prime}$.

For each $\ell \in A$ such that $k_{1} P_{i} \ell P_{i} k_{2}, \sum_{h \in\{1, \cdots, \ell\}} \pi_{i h} \geq \sum_{h \in\{1, \cdots, \ell\}} \hat{\pi}_{i h}=\sum_{h \in\left\{1, \cdots, k_{1}\right\}} \hat{\pi}_{i h}+$ $\sum_{h \in\left\{k_{1}+1, \cdots, \ell\right\}} \hat{\pi}_{i h} \geq \sum_{h \in\left\{1, \cdots, k_{1}\right\}} \pi_{i h}^{\prime}+\sum_{h \in\left\{k_{1}+1, \cdots, \ell\right\}} \pi_{i h}^{\prime}=\sum_{h \in\{1, \cdots, \ell\}} \pi_{i h}^{\prime}$.

Also, since $\hat{\pi}_{i k_{1}}+\hat{\pi}_{i k_{2}}=\pi_{i k_{1}}^{\prime}+\pi_{i k_{2}}^{\prime}, \sum_{h \in\left\{1, \cdots, k_{2}\right\}} \pi_{i h} \geq \sum_{h \in\left\{1, \cdots, k_{2}\right\}} \hat{\pi}_{i h}=\sum_{h \in\left\{1, \cdots, k_{2}\right\} \backslash\left\{k_{1}, k_{2}\right\}} \hat{\pi}_{i h}+$ $\hat{\pi}_{i k_{1}}+\hat{\pi}_{i k_{2}}=\sum_{h \in\left\{1, \cdots, k_{2}\right\} \backslash\left\{k_{1}, k_{2}\right\}} \pi_{i h}^{\prime}+\pi_{i k_{1}}^{\prime}+\pi_{i k_{2}}^{\prime}=\sum_{h \in\left\{1, \cdots, k_{2}\right\}} \pi_{i h}^{\prime}$.

For each $\ell \in A$ such that $k_{2} P_{i} \ell, \sum_{h \in\{1, \cdots, \ell\}} \pi_{i h} \geq \sum_{h \in\{1, \cdots, \ell\}} \hat{\pi}_{i h}=\sum_{h \in\{1, \cdots, \ell\}} \pi_{i h}^{\prime}$. Thus, we conclude that $\pi_{i} R_{i}^{s d} \pi_{i}^{\prime}$.

Next, we prove the implication for the $d l$-extension.

Lemma 2. Let $\mathcal{D}$ be a connected domain satisfying the POF path property. Then for each $m \in \mathbb{N}$, $d l$-within- $m$ strategy-proofness implies $d l$-within- $(m+1)$ strategy-proofness. 
Proof. Let $\mathcal{D}$ be as in the lemma. Let $m \in \mathbb{N}$. Let $\varphi$ be a dl-within-m strategy-proof rule. Let $R \in \mathcal{D}^{N}$ and $i \in N$. Let $R_{i}^{\prime} \in \mathcal{D}$ be such that $d\left(R_{i}, R_{i}^{\prime}\right) \leq m+1$. Let $\pi_{i} \equiv \varphi_{i}(R)$ and $\pi_{i}^{\prime} \equiv \varphi_{i}\left(R_{i}^{\prime}, R_{-i}\right)$. If $d\left(R_{i}, R_{i}^{\prime}\right) \leq m$, then by dl-within-m strategy-proofness, $\pi_{i} R_{i}^{d l} \pi_{i}^{\prime}$. Thus, assume, henceforth, that $d\left(R_{i}, R_{i}^{\prime}\right)=m+1$. Suppose, without loss of generality, that $R_{i}$ is such that $1 P_{i} 2 P_{i} \cdots P_{i} n$ and that $R_{i}^{\prime}$ is such that $k_{1} P_{i}^{\prime} k_{2} P_{i}^{\prime} \cdots P_{i}^{\prime} k_{n}$. We distinguish two cases.

Case 1: $k_{1} \neq 1$.

Since $\mathcal{D}$ satisfies the POF path property, there is $\hat{R}_{i} \in \mathcal{D}$ such that (i) $d\left(R_{i}, \hat{R}_{i}\right)=1$; and (ii) the ranking of object $k_{1}$ is higher according to $\hat{R}_{i}$ than according to $R_{i}$. Let $\hat{\pi}_{i} \equiv \varphi_{i}\left(\hat{R}_{i}, R_{-i}\right)$. Since $m \geq 1$, by $d l$-within-m strategy-proofness, $\pi_{i} R_{i}^{d l} \hat{\pi}_{i}$ and $\hat{\pi}_{i} \hat{R}_{i}^{d l} \pi_{i}$, so that

$$
\begin{aligned}
& \text { either } \quad \text { (i) } \pi_{i}=\hat{\pi}_{i} \\
& \text { or } \quad \text { (ii) for each } \ell \in\left\{1, \cdots, k_{1}-2\right\}, \pi_{i \ell}=\hat{\pi}_{i \ell} \text {; } \\
& \pi_{i, k_{1}-1}>\hat{\pi}_{i, k_{1}-1} ; \text { and } \pi_{i k_{1}}<\hat{\pi}_{i k_{1}} .
\end{aligned}
$$

Further, since $d\left(\hat{R}_{i}, R_{i}^{\prime}\right)=m$, again by dl-within-m strategy-proofness, $\hat{\pi}_{i} \hat{R}_{i}^{d l} \pi_{i}^{\prime}$ and $\pi_{i}^{\prime}\left(R_{i}^{\prime}\right)^{d l} \hat{\pi}_{i}$.

If $\hat{\pi}_{i}=\pi_{i}^{\prime}$, then by $\pi_{i} R_{i}^{d l} \hat{\pi}_{i}, \pi_{i} R_{i}^{d l} \pi_{i}^{\prime}$. Thus, assume, henceforth, that $\hat{\pi}_{i} \neq \pi_{i}^{\prime}$, so that $\hat{\pi}_{i} \hat{P}_{i}^{d l} \pi_{i}^{\prime}$. Now there are four subcases.

Case 1.1: There is $\ell^{*} \in\left\{1, \cdots, k_{1}-2\right\}$ such that (i) for each $\ell \in\left\{1, \cdots, \ell^{*}-1\right\}$, $\hat{\pi}_{i \ell}=\pi_{i \ell}^{\prime}$; and $\left(\right.$ ii) $\hat{\pi}_{i \ell^{*}}>\pi_{i \ell^{*}}^{\prime}$.

Case 1.2: For each $\ell \in\left\{1, \cdots, k_{1}-2\right\}, \hat{\pi}_{i \ell}=\pi_{i \ell}^{\prime}$ and $\hat{\pi}_{i k_{1}}>\pi_{i k_{1}}^{\prime}$.

Case 1.3: For each $\ell \in\left\{1, \cdots, k_{1}-2, k_{1}\right\}, \hat{\pi}_{i \ell}=\pi_{i \ell}^{\prime}$ and $\hat{\pi}_{i, k_{1}-1}>\pi_{i, k_{1}-1}^{\prime}$.

Case 1.4: There is $\ell^{*} \in\left\{k_{1}+1, \cdots, n\right\}$ such that (i) for each $\ell \in\left\{1, \cdots, \ell^{*}-1\right\}$, $\hat{\pi}_{i \ell}=\pi_{i \ell}^{\prime}$; and (ii) $\hat{\pi}_{i \ell^{*}}>\pi_{i \ell^{*}}^{\prime}$.

In Cases 1.1, 1.3, and 1.4, Statement (5) implies that $\pi_{i} P_{i}^{d l} \pi_{i}^{\prime}$. In Case 1.2, it follows that $\hat{\pi}_{i}\left(P_{i}^{\prime}\right)^{d l} \pi_{i}^{\prime}$, in contradiction to $\pi_{i}^{\prime}\left(R_{i}^{\prime}\right)^{d l} \hat{\pi}_{i}$. (Note that if $k_{1}=2$, then Case 1.1 does not apply.) In sum, $\pi_{i} R_{i}^{d l} \pi_{i}^{\prime}$, as desired.

Case 2: There is $h \in\{2, \cdots, n\}$ such that (i) for each $\tilde{h} \in\{1, \cdots, h-1\}, k_{\tilde{h}}=\tilde{h}$; and (ii) $k_{h} \neq h$.

The argument is essentially the same as that in Case 1; only minor changes in notation are needed. The proof is omitted.

Finally, we prove the implication for the ul-extension.

Lemma 3. Let $\mathcal{D}$ be a connected domain satisfying the LOF path property. Then for each $m \in \mathbb{N}$, $u l$-within- $m$ strategy-proofness implies $u$-within- $(m+1)$ strategy-proofness. 
Proof. Let $\mathcal{D}$ be as in the lemma. Let $m \in \mathbb{N}$. Let $\varphi$ be a ul-within-m strategy-proof rule. Let $R \in \mathcal{D}^{N}$ and $i \in N$. Let $R_{i}^{\prime} \in \mathcal{D}$ be such that $d\left(R_{i}, R_{i}^{\prime}\right) \leq m+1$. Let $\pi_{i} \equiv \varphi_{i}(R)$ and $\pi_{i}^{\prime} \equiv \varphi_{i}\left(R_{i}^{\prime}, R_{-i}\right)$. If $d\left(R_{i}, R_{i}^{\prime}\right) \leq m$, then by ul-within-m strategy-proofness, $\pi_{i} R_{i}^{u l} \pi_{i}^{\prime}$. Thus, assume, henceforth, that $d\left(R_{i}, R_{i}^{\prime}\right)=m+1$. Suppose, without loss of generality, that $R_{i}$ is such that $1 P_{i} 2 P_{i} \cdots P_{i} n$ and that $R_{i}^{\prime}$ is such that $k_{1} P_{i}^{\prime} k_{2} P_{i}^{\prime} \cdots P_{i}^{\prime} k_{n}$. We distinguish two cases.

Case 1: $k_{n} \neq n$.

Since $\mathcal{D}$ satisfies the LOF path property, there is $\hat{R}_{i} \in \mathcal{D}$ be such that (i) $d\left(R_{i}, \hat{R}_{i}\right)=1$; and (ii) the ranking of object $k_{n}$ is lower according to $\hat{R}_{i}$ than according to $R_{i}$. Let $\hat{\pi}_{i} \equiv \varphi_{i}\left(\hat{R}_{i}, R_{-i}\right)$. Since $m \geq 1$, by ul-within-m strategy-proofness, $\pi_{i} R_{i}^{u l} \hat{\pi}_{i}$ and $\hat{\pi}_{i} \hat{R}_{i}^{u l} \pi_{i}$, so that

$$
\begin{aligned}
& \text { either (i) } \pi_{i}=\hat{\pi}_{i} \\
& \text { or } \quad \text { (ii) for each } \ell \in\left\{n, n-1, \cdots, k_{n}+2\right\}, \pi_{i \ell}=\hat{\pi}_{i \ell} \text {; } \\
& \quad \pi_{i, k_{n}+1}<\hat{\pi}_{i, k_{n}+1} \text {; and } \pi_{i k_{n}}>\hat{\pi}_{i k_{n}} .
\end{aligned}
$$

Further, since $d\left(\hat{R}_{i}, R_{i}^{\prime}\right)=m$, again by ul-within-m strategy-proofness, $\hat{\pi}_{i} \hat{R}_{i}^{u l} \pi_{i}^{\prime}$ and $\pi_{i}^{\prime}\left(R_{i}^{\prime}\right)^{u l} \hat{\pi}_{i}$.

If $\hat{\pi}_{i}=\pi_{i}^{\prime}$, then by $\pi_{i} R_{i}^{u l} \hat{\pi}_{i}, \pi_{i} R_{i}^{u l} \pi_{i}^{\prime}$. Thus, assume, henceforth, that $\hat{\pi}_{i} \neq \pi_{i}^{\prime}$, so that $\hat{\pi}_{i} \hat{P}_{i}^{u l} \pi_{i}^{\prime}$. Now there are four subcases.

Case 1.1: There is $\ell^{*} \in\left\{n, n-1, \cdots, k_{n}+2\right\}$ such that $(i)$ for each $\ell \in\left\{n, n-1, \cdots, \ell^{*}+1\right\}$, $\hat{\pi}_{i \ell}=\pi_{i \ell}^{\prime}$; and (ii) $\hat{\pi}_{i \ell^{*}}<\pi_{i \ell^{*}}^{\prime}$.

Case 1.2: For each $\ell \in\left\{n, n-1, \cdots, k_{n}+2\right\}, \hat{\pi}_{i \ell}=\pi_{i \ell}^{\prime}$ and $\hat{\pi}_{i k_{n}}<\pi_{i k_{n}}^{\prime}$.

Case 1.3: For each $\ell \in\left\{n, n-1, \cdots, k_{n}+2, k_{n}\right\}, \hat{\pi}_{i \ell}=\pi_{i \ell}^{\prime}$ and $\hat{\pi}_{i, k_{n}+1}<\pi_{i, k_{n}+1}^{\prime}$.

Case 1.4: There is $\ell^{*} \in\left\{k_{n}-1, k_{n}-2, \cdots, 1\right\}$ such that (i) for each $\ell \in\left\{n, n-1, \cdots, \ell^{*}+1\right\}$, $\hat{\pi}_{i \ell}=\pi_{i \ell}^{\prime}$; and $(i i) \hat{\pi}_{i \ell^{*}}<\pi_{i \ell^{*}}^{\prime}$.

In Cases 1.1, 1.3, and 1.4, Statement (6) implies that $\pi_{i} P_{i}^{u l} \pi_{i}^{\prime}$. In Case 1.2, it follows that $\hat{\pi}_{i}\left(P_{i}^{\prime}\right)^{u l} \pi_{i}^{\prime}$, in contradiction to $\pi_{i}^{\prime}\left(R_{i}^{\prime}\right)^{u l} \hat{\pi}_{i}$. (Note that if $k_{n}=n-1$, then Case 1.1 does not apply.) In sum, $\pi_{i} R_{i}^{u l} \pi_{i}^{\prime}$, as desired.

Case 2: There is $h \in\{n, \cdots, 2\}$ such that (i) for each $\tilde{h} \in\{n, n-1, \cdots, h+1\}, k_{\tilde{h}}=\tilde{h}$; and (ii) $k_{h} \neq h$.

The argument is essentially the same as that in Case 1; only minor changes in notation are needed. The proof is omitted. 


\section{B Appendix: $d l$-strategy-proofness of the Serial Rule}

In this appendix, we prove part (1) of Theorem 7. By Theorem 1, it suffices to show that the serial rule, denoted $\boldsymbol{S}$, is dl-adjacent strategy-proof. Let $R \in \mathcal{R}(A)^{N}$ and $i \in N$. Let $R_{i}^{\prime} \in \mathcal{R}(A)$ be adjacent to $R_{i}$. Without loss of generality, assume that $1 P_{i} 2 P_{i} \cdots P_{i} n$. Let $k \in A$ be such that $(k+1) P_{i}^{\prime} k$. Let $\pi \equiv S(R)$ and $\pi^{\prime} \equiv S\left(R_{i}^{\prime}, R_{-i}\right)$. Suppose, by contradiction, that $\pi_{i}^{\prime} P_{i}^{d l} \pi_{i}$.

Consider the simultaneous consumption algorithm applied to the economy $R$. We use the following notation throughout the proof. ${ }^{21}$ For each $(\ell, t) \in A \times \mathbb{R}_{+}$, let $\boldsymbol{N}(\boldsymbol{\ell}, \boldsymbol{t})$ be the set of agents who consume object $\ell$ at time $t$; i.e., for each $\ell \in A$ and each $t \in \mathbb{R}_{+}$such that $t \in\left[t^{s-1}, t^{s}\right)$ for some $s \in \mathbb{N}, N(\ell, t) \equiv N^{*}\left(R, N^{s-1}, A^{s-1}, \ell\right)$. Note that $N(\ell, t)$ may be empty. Also, for each $\ell \in A$, let $\boldsymbol{t}(\ell)$ be the time at which object $\ell$ is exhausted; i.e., $t(\ell) \equiv \sup \left\{t \in \mathbb{R}_{+}: N(\ell, t) \neq \emptyset\right\}$. Let $\boldsymbol{t}_{\mathbf{0}} \equiv \max _{\ell \in\{1, \cdots, k-1\}} t(\ell)$ if $k \neq 1$; and $\boldsymbol{t}_{\mathbf{0}} \equiv 0$ otherwise. Define $N^{\prime}(\ell, t), t^{\prime}(\ell)$, and $t_{0}^{\prime}$ similarly for the economy $\left(R_{i}^{\prime}, R_{-i}\right)$.

It is easy to see that for each $\ell \in\{1, \cdots, k-1\}, \pi_{i \ell}=\pi_{i \ell}^{\prime}$ and $t_{0}=t_{0}^{\prime}$. Now we proceed in four steps.

Step 1: $t(k) \leq t^{\prime}(k)$.

In the algorithm that determines $\pi$, agent $i$ consumes object $k$ during the interval $\left[t_{0}, t(k)\right)$, so that $\pi_{i k}=t(k)-t_{0}$. On the other hand, in the algorithm that determines $\pi^{\prime}$, agent $i$ consumes object $k$ during a subinterval of $\left[t_{0}, t^{\prime}(k)\right)$. Thus, if $t(k)>t^{\prime}(k)$,

$$
\pi_{i k}^{\prime} \leq t^{\prime}(k)-t_{0}<t(k)-t_{0}=\pi_{i k}
$$

contradicting that $\pi_{i}^{\prime} P_{i}^{d l} \pi_{i}$.

Step 2: For each $t \in\left[t_{0}, t(k)\right), N(k, t) \backslash\{i\}=N^{\prime}(k, t) \backslash\{i\}$.

We only show that for each $t \in\left[t_{0}, t(k)\right), N(k, t) \backslash\{i\} \subseteq N^{\prime}(k, t) \backslash\{i\}$; the reverse inclusion can be proved similarly. Suppose, by contradiction, that there are $\hat{t} \in\left[t_{0}, t(k)\right), j \in N \backslash\{i\}$, and $\hat{\ell} \in A \backslash\{k\}$ such that $j \in N(k, \hat{t}) \cap N^{\prime}(\hat{\ell}, \hat{t})$. We proceed in three steps.

Step 2.1: Let $B \equiv\left\{\ell \in A \backslash\{k\}: t(\ell)<t^{\prime}(\ell)\right\}$ and $h \equiv \arg \min _{\ell \in B} t(\ell)$. Then $B \neq \emptyset$ and $t(h)<t(k)$.

Since $\hat{t}<t(k) \leq t^{\prime}(k)$ and $j \in N^{\prime}(\hat{\ell}, \hat{t}), \hat{\ell} P_{j} k$ and $\hat{t}<t^{\prime}(\hat{\ell})$. Also, since $j \in N(k, \hat{t}), t(\hat{\ell}) \leq \hat{t}$. Thus, $\hat{\ell} \in B$, so that $B \neq \emptyset$. Further, since $t(h) \leq t(\hat{\ell}) \leq \hat{t}<t(k), t(h)<t(k)$.

Step 2.2: There are $\bar{t} \in\left[t_{0}, t(h)\right)$ and $j^{\prime} \in N$ such that $j^{\prime} \in N(h, \bar{t}) \cap N^{\prime}(h, \bar{t})^{c}$.

\footnotetext{
${ }^{21}$ Some of the notations are borrowed from Bogomolnaia and Moulin (2001).
} 
Suppose, by contradiction, that for each $t \in\left[t_{0}, t(h)\right), N(h, t) \subseteq N^{\prime}(h, t)$. Since $t(h)<t^{\prime}(h)$,

$$
\begin{aligned}
1 & =\int_{0}^{t^{\prime}(h)}\left|N^{\prime}(h, t)\right| d t \\
& =\int_{0}^{t_{0}}\left|N^{\prime}(h, t)\right| d t+\int_{t_{0}}^{t(h)}\left|N^{\prime}(h, t)\right| d t+\int_{t(h)}^{t^{\prime}(h)}\left|N^{\prime}(h, t)\right| d t \\
& >\int_{0}^{t_{0}}|N(h, t)| d t+\int_{t_{0}}^{t(h)}|N(h, t)| d t \\
& =\int_{0}^{t(h)}|N(h, t)| d t \\
& =1
\end{aligned}
$$

where the inequality follows from the fact that there is $\tilde{t} \in\left[t(h), t^{\prime}(h)\right)$ such that for each $t \in$ $\left[\tilde{t}, t^{\prime}(h)\right), N^{\prime}(h, t) \neq \emptyset$. This is a contradiction.

Step 2.3: Let $h^{\prime} \in A$ be such that $j^{\prime} \in N^{\prime}\left(h^{\prime}, \bar{t}\right)$. Then $h^{\prime} \in B$ and $t\left(h^{\prime}\right)<t(h)$, contradicting our choice of $h$.

By Steps 2.1 and 2.2, $\bar{t}<t(h)<t(k)$. Also, in the algorithm that determines $\pi$, agent $i$ consumes object $k$ during $\left[t_{0}, t(k)\right)$. Thus, $j^{\prime} \neq i$. Since $\bar{t}<t(h)<t^{\prime}(h)$ and $j^{\prime} \in N^{\prime}\left(h^{\prime}, \bar{t}\right), h^{\prime} P_{j^{\prime}} h$ and $\bar{t}<t^{\prime}\left(h^{\prime}\right)$. Since $j^{\prime} \in N(h, \bar{t})$, the fact that $h^{\prime} P_{j^{\prime}} h$ implies that $t\left(h^{\prime}\right) \leq \bar{t}$. Thus, $h^{\prime} \in B$. Moreover, $t\left(h^{\prime}\right) \leq \bar{t}<t(h)$.

Step 3: (i) $t(k)=t^{\prime}(k)$; (ii) $\pi_{i k}=\pi_{i k}^{\prime}$; and (iii) in the algorithm that determines $\pi^{\prime}$, agent $i$ consumes object $k$ during the interval $\left[t_{0}, t^{\prime}(k)\right)=\left[t_{0}, t(k)\right)$.

To show (i), suppose, by contradiction, $t(k) \neq t^{\prime}(k)$. By Step $1, t(k)<t^{\prime}(k)$. Note that for each $t \in\left[0, t_{0}\right), N(k, t)=N^{\prime}(k, t)$. Thus,

$$
\begin{aligned}
1-\pi_{i k} & =\int_{0}^{t(k)}|N(k, t) \backslash\{i\}| d t \\
& =\int_{0}^{t_{0}}|N(k, t) \backslash\{i\}| d t+\int_{t_{0}}^{t(k)}|N(k, t) \backslash\{i\}| d t \\
& =\int_{0}^{t_{0}}\left|N^{\prime}(k, t) \backslash\{i\}\right| d t+\int_{t_{0}}^{t(k)}\left|N^{\prime}(k, t) \backslash\{i\}\right| d t \\
& <\int_{0}^{t_{0}}\left|N^{\prime}(k, t) \backslash\{i\}\right| d t+\int_{t_{0}}^{t(k)}\left|N^{\prime}(k, t) \backslash\{i\}\right| d t+\int_{t(k)}^{t^{\prime}(k)}\left|N^{\prime}(k, t) \backslash\{i\}\right| d t \\
& =\int_{0}^{t^{\prime}(k)}\left|N^{\prime}(k, t) \backslash\{i\}\right| d t \\
& =1-\pi_{i k}^{\prime},
\end{aligned}
$$

where the third equality follows from Step 2 and the inequality from the fact that there is $\tilde{t} \in$ 
$\left[t(k), t^{\prime}(k)\right)$ such that for each $t \in\left[\tilde{t}, t^{\prime}(k)\right), N^{\prime}(k, t) \backslash\{i\} \neq \emptyset$. Thus, $\pi_{i k}>\pi_{i k}^{\prime}$, contradicting that $\pi_{i}^{\prime} P_{i}^{d l} \pi_{i}$

To show (ii) and (iii), recall that in the algorithm that determines $\pi^{\prime}$, agent $i$ consumes object $k$ during a subinterval of $\left[t_{0}, t^{\prime}(k)\right)$. Since $t(k)=t^{\prime}(k)$ and $\pi_{i k}^{\prime} \geq \pi_{i k}$, agent $i$, in fact, consumes object $k$ during the interval $\left[t_{0}, t^{\prime}(k)\right)=\left[t_{0}, t(k)\right)$. Thus, $\pi_{i k}^{\prime}=t^{\prime}(k)-t_{0}=\pi_{i k}$.

Step 4: Concluding.

If $t(k) \leq t_{0}$, then object $k$ is not available at time $t_{0}$ in each of the two algorithms that determine $\pi$ and $\pi^{\prime}$, respectively. Thus, the two algorithms coincide. If $t(k)>t_{0}$, then by (iii) in Step 3 , $t^{\prime}(k+1) \leq t_{0}$ and object $k+1$ is not available at time $t_{0}$ in each of the two algorithms. Thus, the two algorithms again coincide. In either case, $\pi=\pi^{\prime}$, in contradiction to $\pi_{i}^{\prime} P_{i}^{d l} \pi_{i}$.

\section{References}

[1] Abdulkadiroğlu, A., and T. Sönmez, 1999, "House allocation with existing tenants", Journal of Economic Theory, 88, 233-260.

[2] Abdulkadiroğlu, A., and T. Sönmez, 2003a, "Ordinal efficiency and dominated sets of assignments", Journal of Economic Theory, 112, 157-172.

[3] Abdulkadiroğlu, A., and T. Sönmez, 2003b, "School choice: a mechanism design approach", American Economic Review, 93, 729-747.

[4] Alcalde, J., 2013, "Ransom housing with existing tenants", mimeo.

[5] Alcalde, J., and J. A. Silva-Reus, 2013, "Allocating via priorities", mimeo.

[6] Aziz, A., F. Brandl, and F. Brandt, 2014, "On the incompatibility of efficiency and strategyproofness in randomized social choice", mimeo.

[7] Birkhoff, G., 1946, "Three observations on linear algebra", Revi. Univ. Nac. Tucuman, ser A $5,147-151$.

[8] Bogomolnaia, A., 2012, "Random assignment: redefining the serial rule", mimeo.

[9] Bogomolnaia, A., and E. J. Heo, 2012, "Probabilistic assignment of objects: characterizing the serial rule", Journal of Economic Theory, 147, 2072-2082.

[10] Bogomolnaia, A., and H. Moulin, 2001, "A new solution to the random assignment problems", Journal of Economic Theory, 100, 295-328. 
[11] Budish, E., Y.-K. Che, F. Kojima, and P. Milgrom, 2013, "Designing random allocation mechanisms: theory and applications", American Economic Review, 103, 585-623.

[12] Carroll, G., 2010, "An efficiency theorem for incompletely known preferences", Journal of Economic Theory, 145, 2463-2470.

[13] Carroll, G., 2012, "When are local incentive constraints sufficient?", Econometrica, 80, 661686.

[14] Che, Y.-K., and F. Kojima, 2010, "Asymptotic equivalence of probabilistic serial and random priority mechanisms", Econometrica, 78, 1625-1672.

[15] Chipman, J. S., 1960, "The foundations of utility", Econometrica, 28, 193-224.

[16] Foley, D., 1967, "Resource allocation and the public sector", Yale Economic Essays, 7, 45-98.

[17] Gibbard, A., 1977, "Manipulation of schemes that mix voting with chance", Econometrica, $45,665-681$.

[18] Haeringer, G., and H. Halaburda, 2014, "Monotone strategy-proofness", mimeo.

[19] Hashimoto, T., D. Hirata, O. Kesten, M. Kurino, M. U. Ünver, 2014, "Two axiomatic approaches to the probabilistic serial mechanism", Theoretical Economics, 9, 253-277

[20] Hausner, M., 1954, "Multidimensional utilities", in Decision Processes (R. M. Thrall, C. H. Coombs, R. L. Davis, Eds.), Wiley, New York.

[21] Hylland, A., and R. Zeckhauser, 1979, "The efficient allocation of individuals to positions", Journal of Political Economy, 87, 293-314.

[22] Kasajima, Y., 2013, "Probabilistic assignment of indivisible goods with single-peaked preferences", Social Choice and Welfare, 41, 203-215.

[23] Katta, A.-K., and J. Sethuraman, 2006, "A solution to the random assignment problem on the full preference domain", Journal of Economic Theory, 131, 231-250.

[24] Kemeny, J. G., 1959, "Mathematics without Numbers", Daedalus, 577-591.

[25] Kemeny, J. G., and J. L. Snell, 1962, Mathematical Models in the Social Sciences, Blaisdell, New York.

[26] Kesten, O., 2009, "Why do popular mechanisms lack efficiency in random environments?", Journal of Economic Theory, 144, 2209-2226. 
[27] Kojima, F., 2009, "Random assignment of multiple indivisible objects", Mathematical Social Sciences, 57, 134-142.

[28] Liu, Q., and M. Pycia, 2012, "Ordinal efficiency, fairness, and incentives in large markets", mimeo.

[29] Manea, M., 2008, "A constructive proof of the ordinal efficiency welfare theorem", Journal of Economic Theory, 141, 276-281.

[30] Manea, M., 2009, "Asymptotic ordinal inefficiency of random serial dictatorship", Theoretical Economics, 4, 165-197.

[31] McLennan, A., 2002, "Ordinal efficiency and the polyhedral separating hyperplane theorem", Journal of Economic Theory, 105, 435-449.

[32] von Neumann, J., 1953, "A certain zero-sum two-person game equivalent to the optimal assignment problem", in Contributions to the Theory of Games, 2 (H. W. Kuhn and A. W. Tuckers, Eds.), Princeton University Press, Princeton, New Jersey.

[33] Saban, D., and J. Sethuraman, 2013, "A note on object allocation under lexicographic preferences", mimeo.

[34] Sato, S., 2013a, "A sufficient condition for the equivalence of strategy-proofness and nonmanipulability by preferences adjacent to the sincere one", Journal of Economic Theory, 148, 259-278.

[35] Sato, S., 2013b, "Strategy-proofness and the reluctance to make large lies: the case of weak orders", Social Choice and Welfare, 40, 479-494.

[36] Schulman, L. J., and V. V. Vazirani, 2012, "Allocation of divisible goods under lexicographic preferences", mimeo.

[37] Tinbergen, I., 1953, Redelijke Inkomensverdeling, N. V. DeGulden Pers, Haarlem, The Netherlands, 2nd edition.

[38] Yilmaz, O., 2009, "Random assignment under weak preferences", Games and Economic Behavior, 66, 546-558.

[39] Yilmaz, O., 2010, "The probabilistic serial mechanism with private endowments", Games and Economic Behavior, 69, 475-491. 
[40] Zhou, L., 1990, "On a conjecture by Gale about one-sided matching problems", Journal of Economic Theory, 52, 123-135. 